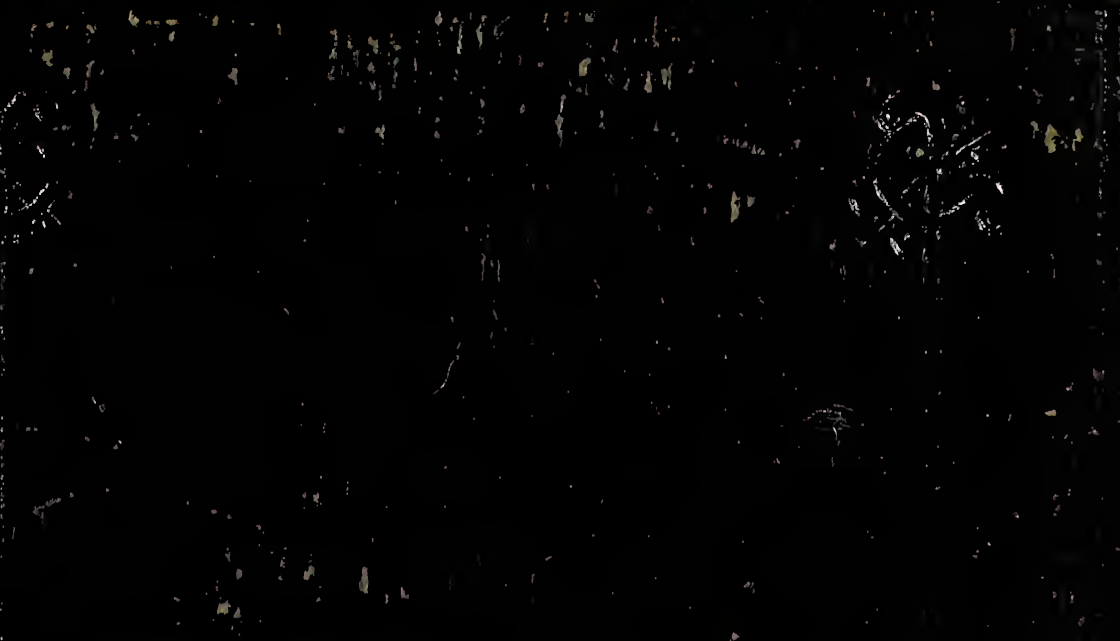

\title{
MATWHEWNIM TAAISKT.
}

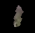

\section{MATTHEW,}

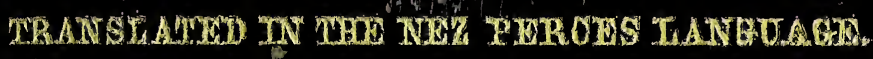

1
4
4

a 



$$
\text { Sang: }
$$







\section{MATTHEWNIM TAAISKT.}

THE GOSPEL ACCORDING TO it

\subsection{1 \\ MATTHEW,}

TRANSLATED INTO THE NEZ PERCES LANGUAGE, BY REV. H. H. SPALDING, MISSIONARY OF THE A.B. C. F. M.

NEW YORK:

AMERICAN BIBLE SOCIETY, INSTITUTED IN THE YEAR MDCCCXVI.

1871. 


$$
\begin{aligned}
& P M 2019 \\
& 27 B 56 \\
& 1871
\end{aligned}
$$

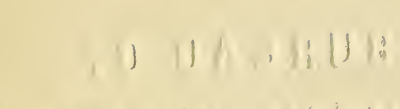

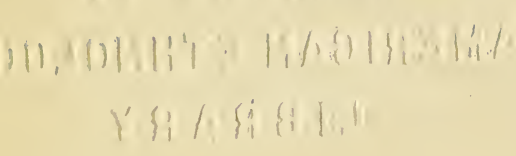




\section{MATTHEWNIM TAAISKT.}

WANAHNA I.

TIMASH hiwash Jesus Christpkinih wiaut1 sath kuph. Davidnim miahs awaka Jesus Christ, Abrahamnim miahs awaka David. 2 Abrahainnim miahs autsama Isaac; Isaacnim miahs autsama Jacob; Jacobnim mamaias autsama Judas wak askama;

3 Judasnim autsama mamaias Phares wah Zara, Tharmapkinih; Pharesnim miahs autsama Esrom ; Esromnim miahs autsama Aram;

4 Aramniun miahs autsama Aminadab; Aminadabnim miahs autsama Naason; Naasonm miahs autsama Salmon;

5 Salmonm miahs autsama Booz Rachabkinih; Booznim miahs autsama Obed Ruthpkinih; Obednim miahs autsama Jesse;

6 Jessenim miahs autsama David, Miohat; Davidnim Miohatom miahs autsama Solomon, ka yoh awaka iwapna Urianm, kunimpkinih;

7 Solomon miahs autsama Roboam; Roboamnim miahs autsama Abia; Abianm miahs autsama Asa;

8 Asanm miahs autsama Josaphat; Josaphatom miahs autsama Joram; Joramnim miahs autsama Ozias;

9. Oziasnim miahs autsama Joatham; Joa- 
thamnim miahs autsama Achaz; Achaznim miahs autsama Ezekias;

10 Ezekiasnim miahs autsama Manases; Manasesnmi miahs autsama Amon; Amonnim miahs autsama Josias ;

11 Josiasnim mamaias autsama Jechonias wak askama, ka kaua Babylonpa panahnasankika immuna.

12 Ka kaua panahpaiksankika Babylonpa immuna, kaua Jekoniasnim miahs autsama Salathiel ; Salathielm miahs autsama Zorobabel ;

13 Zorobabelm miahs autsama Abiud; Abiudnim miahs autsama Azor;

14 Azornm miahs autsama Sadoc; Sadocnim miahs autsama Achim, Achimnim miahs autsama Eliud;

15 Eliudnim miahs autsama Eleazar, Eleazarnim miahs autsama Matthan; Matthanm miahs autsama Jacob;

16 Jacobnim miahs autsama Joseph; mat ata yoh Marynm hama autsatatashana, kunih hiutsama Jesus, kanm yoh unikshi Christ.

17 Kunki uyikala wiapiim Abrahamphkinih hiutsama putimpiim wah pilapipiim Davidph kaua Davidphkinih uyikala wiapiim hiutsama putim piim wah pilapipiim watkunikashph Babylonph; kaua Babylonphkinih wotkuñikashphkinih uyikala wiapiim hutsama putimpiim wah pilapipiim Christph.

18 Kush Jesus Christnim witsat autsaya ip- 
nim pika Mary wako Josephnim iwapna awaka tamaluitki, kos watu pikioshina, matu wako awaka ilutpa Holy Ghostki.

19 Matu ipnim hama Joseph hiwaka tukuh, kunki hinakshana ka watu tamapaiks, tamapaikaiai auyuisa Maryna.

20 Kos hitimiusana, ki mat kaua naks Angel Lordpkinih patkaikoma, pashapahwakima, kaua patnueya Joseph, Davidnim miahs ituain himaisa Maryna im wapnaph, inpukinih; Holy Ghostki ilotpa hiwash.

21 Kaua haswala pakaiatatasha, kaua a uniktatasha Jesus; atka ipnim titokan hinatsnakahtatasha immam patokashwitkinih.

22 Kinki wiakutki Lordnim tsekin imatsinpunki atsanima, ka yoh hitsehna;

23 "Mitsimith! kos nakspa timaipa ilotpa hiutsayu, kaua pakaiatatasha miashna, kaua kunia puniktatashi Emmanuel, tamanitki, Godhin kia washi."

24 Ka kaua Joseph hiwahna, ka kush angelm patanuama Lordphkinih kaua kush hikuya, kunki iwapnin hipautsaya:

25 Matu kos watu patautuasina, kaua yohnikos miahsna pakaisana, haswalna: kaua kunia punika Jesus.

\section{WANAHNA II.}

T. A kaua Jesus huitsaya Judeanm Beth1 lehempa, ka kaua Herod himiohatowi- 
shana, kimat kaua Jerusalempa papainoshana wiwapsuhnim tinatitkinikaikinih,

2 Pasana, ma hiwash, ka ipi Jewsnim Miohat apawitsas; wako nun ipna hetseyu apahnanima tinahtitkinikaikinih, kunki ipna ،apakantamah.

$3 \mathrm{Ka}$ kaua miohat Herod hinashmitsia kaua hitahtakatna Jerusalempuhina.

4 Kaua titokana titalamionawatona wah titimanawatona uyikalona hinashapaamka, kaua hinashapnia mina Christnim ush wisatash ?

5 Kaua pasana Judeanm Bethlehempa: atka kush imatsinpunm hitimana;

$6 \mathrm{Im}$ Bethlehem, Judeanm wataspa, watu awash laimut Judapama mimiohatopkinih atka imkinih huitsatatasha naks miohat, kauakunim ina titokan Israelpuna himiohatoiyoaitaaisha.

$7 \mathrm{Ka}$ kaua Herod ipnashtakin hinashmuna wiwapsuhna kaua lauit hinashapnia maua hetseyu apahnanima?

8 Kaua hinashapawihnana immuna Bethlehemph ; hinashna kutathku Lauit apawit kuskusana miahsna; kapam kaua apaiyaktanu kaua atk ina patamtainukum, kaua inka ipna akantanu.

$9 \mathrm{Ka}$ kaua miohatona pamsia kaua hipakuya; ki mat, ka kunia paksina hetseyuna, tinahtitkinikaikinih, kunimtita hinatsnoktianya; kuskusam miahsnim kunmakam patkatalkoma.

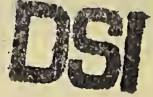


$10 \mathrm{Ka}$ kaua hetseuna paksina, kaua hipaqailtamauna.

11 Ka kaua initpa hipaasima, kaua paksana kuskusana miahsna, wah ipna pika Maryna, kaua patqoikokalikoshana potahskshana Ipna, kaua titahsna hipankapkuika, yoh ka initahs aushina, kaua yokopi panishana; Gold, Frankinsens wah marh.

$12 \mathrm{Ki}$ mat shapahwakaiih hinashtanuema Godnim, wat mat Herodna apakiuyu, kunki immam watashph nahshapa iskitpa hipaskilitoka.

$13 \mathrm{Ka}$ kaua hipaskilina, ki mat naks angel Lordpkinih patkaikuma Josephna pashapahwakima, pana, a hipawiaitataisham kuskus miahs wapsiaunash; kunki wakin, kuskusana miahsna pikin auniph anatsnahwilalikin Egypt, kuno ath pautsatanu kos In a hinu.

14 Kaua hiwahna kaua kuskusana miashna panpa pikahina kaua hitaunahwuina.

15 Kaua kanu hiutsaya oko ka kaua Herod hitnuhna. Kunki hitsanima yoh ka awaka sekin Lordnim imatsinpunki; pasana In miahs Egyptkinih muna.

$16 \mathrm{Ka}$ kaua Herod hinashsukuana atak in hipatamolamia kaua hikatamna, kaua hinashapopsiautana Bethlehemph wah lilaikinph, kapam kuna apupaiyahna wiwapsuhnim himtakitki. 
17 Jeremynim imatsinpunm tsekin, kaua hitsanima yoh ka pasana.

18 Ramapa pamsishina, komph, wiph, ilalimhimiuph, Rachel hilalimhimiuna mamaiaski atka saiau awaka kunki watu manama shapushaunash hiwaka.

$19 \mathrm{Ka}$ kaua Herod hitnuhna, ki mat kaua naksnim angelm Lordphkinih patkaikuma Josephna pashapahwakima,

20 Panima ka immam kuskusana miahsna popapawisheka imam wako hipatnukin; kunki auniph kuskusana miahsna pikin, kauo wihnah Israelm watashph.

21 Kaua hiushakaika, kaua kuskusna miahsna panpa pikahina, kaua hipakuma Israelm watashpa.

22 Matu kaua hinashmitsia, ata Arkelas taklai pishitpkinih Herodkinih, himiohatoisha Judeana, kunki Godnim tamaluitki hiwakitki patamolamia Galileeph:

23 Kaua papainoya nakspa tauyanikashpa unikshana Nazareth, kaua kuna hitauyanika; kunki hitsanima haimatsinpunm tsekin, ka yoh huyahtsehnanihna, ipna puniksapinmitatasha Nazarethpu.

\section{WANAHNA III.}

IT AUATITA John Baptainawat hipaina 1 Judeapa, hinashtamtainaiika mimahsha. miih. 
2 Kaua hinashna imamatamaskalikith, kia hikakamnisham akamkinikom inakanikitpim.

$3 \mathrm{Ki}$ wash ka ipna Esaiasnim imatsinpunm panaishana, pasana naksnim mimahshamiih hinashwiasa Lordna kiaiikash onianith, ipna iskit asapatkukanith.

4 Ipnimtita John awaka kamelm tahainim shamh; kaua itpashnim shamawash awaka; hipt awaka shahahnonm wah kahasinm.

5 Kaua ipna pakiushana Jerusalempum, wah uyikala Judeapum, wah Jordan laikinkinih.

6 Kaua immam mitsitpashwit imamatamapaikanitki imamashapabaptaisina Johnph Jordanpa.

7 Ka kaua hinashahna ilahniwana Pharisepuna wah Sadusepuna immamashapabaptaitama, kaua hinashna ima ath washi tukumutaliktkakan, ishinm taskaukitki ath hishapaulaliksa wakainishpkinih?

8 Waya inamashapahnanimth imam wiakut imamatamaskalikitkinih.

9 Wat mat inamanaku nunimsim pisht hiwam Abraham; ath hisa kupam Godnim ilatuashimaiwitki pashapautsatah Abrahamnim mamaias pishwanm.

10 Wako panika wawianasna taulikinm shukutpa: kunki ka kalanim saiau titahs timanit autsatatasha kunia pokuktatasha shapalunash. 
11 Inaki In ath imuna baptaisa kushki imamatamaskaliktash: matu Inmhalih naks hiwahyam, kunim ina hikatosksha kapshiki, watu manama inim inalapkiauki inakihnikash ipna ilapkat; kunim imuna ath hibaptaitatasha Holy Ghostki wah alaki.

12 Wako hinaptain wapuhpuhnash, kunki lauit ipnim shapatulahimkinwash hiwapuhpuhtatasha; kaua lalahsim hiniktatasha inikashpa; kau taklai lahlakasna pashapalutatasha ilatahashshimaiki alaki.

13 Kaua Jesus hipainima Galileepkinih Jordanpa Johnpa, ipnashapabaptaitama ipnimpki.

$14 \mathrm{Ki}$ mat John patatka ipna pana, matu in wash inashapabaptainash imimpki, imat inimph kuma?

15 Kaua Jesusnim pamstakanpa, pana sauin kush kutash awash : nunim washi tuihnash uyikalana tukuhna wiakuna. Kunki hibaptaina.

16 Kaua baptainakatpa Jesus kunainih hitkeya kushpkinih, ki mat ipna (Johna) patamahalpoına akamkinikai, kaua pahnanya Godna Spirit, ka kush kuinu patkainakuma ipna puyalatiakima;

17 Ki mat kaua akamkinikaikinih hitsehnima, pana ki wash inim miahs hataunih, kinki Inim aishnikit wash. 


\section{WANAHNA IV.}

T AUA Spiritnim Jesusna pashapawihnana 11 mimahshamka, shapatahinauitash devilki.

2 Kaua pilaptisikiu hiutsama hipaiai sikatinhina, kaua haiakin autsaya.

3 Kaua tahinauitipasnim papainoya, pana kam ikuin Godnim Miahs waku kaua ashapautsaih kipt kinm pishwanm.

$4 \mathrm{Ki}$ mat kana pamstakanpa, pana wako timanin hiwash watu mish pakaskisim wiapiimnash titokan, kala uyikalaki wiatseki Godnim himkinih.

5 Kaua devilm panahnana hautnish tauyanikash kaua ipna ipnoyiyaukinwashnim ininm sukuitalampa pashaushalikasaya,

6 Kaua pana kam ikuin God nim Miahs wa$\mathrm{ku}$, kaua imatolahna: matu $\mathrm{ku}$ timanin hiwash yoh ka ush haangel hinashwaiyimtatasha imwasatki hnashapanakaniktatasham immam ipshushki kainain im wahuhshtaiku ahwa pishwaph.

7 Kaua Jesusnim pana ipna, hiwash hanaka timanin, wat mat Lordna imim Godna atahinauiyu.

8 Kalla naksiph kaiihph mahshamka devilum panahqoimkika ipna, kaua pashapahna uyikashlih wiwatash titauyanikash, sisiskeishina laam. 
9 Kaua ipna pana kam ina okomolikutatasham, kam inimph imoyiyauktatasham, ka kala hiwash kala a initatasha laam.

10 Kaua Jesusnim ipna pana niwihnam Tahinauiat: wako timanin hiwash, Lordna imim Godna otahsku a ipnasim atqaluku.

11 Kaua devilum panwihnana ipna, ki mat kaua haangelm papainoya, palapayutama ipna.

$12 \mathrm{Ka}$ kaua Jesus pasukuana wako John tulailakin shaktishpa initpa, Galileeph kaua hipakuya.

13 Kaua Nazarethna panwihnana, kaua Capernaumpa hipaina kaua kuna hitauyanika, ka yoh hiwaka iwatam laikin, Zabulon laikin wah Nephthalim laikin:

14 Hishapatsatamah yoh ka hitsehnima Esaianm imatsinpunm, hisana.

15 Zabulonm watash wah Naphthalimnim watash iwatamlaikinpa, Jordan kumkunih Gentilm Galilee.

$16 \mathrm{Ka}$ imma titokan hiwitauyasina shaktishpa immam ata wako paksana himakesna ilakauina: ka imam hiwitauyasina tinukinm watashpa, tinukinm shaktisha immuna hinatslakauyosha.

17 Kaua kunih Jesus hinashtamtainaiika, hinashnaiika imamatamaskalikith imam mitsitpashwitkinih: wako kia hikakamnisham akamkinikom inakanikitpim. 
18 Galileenm iwatamlaikin hiwahyana Jesus, kaua kunih hinashahna lapuana askapin Simon wanikin Peter wak ipniın askap Andrew, hitakashina iwatampa: lilauwaptis hiushina.

19 Kaua hinashna tuihniinth ina, kos ath shapatakelptatasha titokan.

20 Kaua kunmainih panwihnasana takash, kaua patuiksana ipna.

21 Kaua kunih hikuya, kaua hanaka hinashahnakauna, lapuana askapin, James wah John, Zebedeenm imamaias, liashpa pishthinahna, ata hilaulimshina takash, kaua hinashmuna.

22 Kaua kunmainih panwihnasana pisht, wah liash kaua patuiksana ipna.

23 Kaua Jesus uyikashlih Galilee hikiaiika ipnoyiyaukinwashpa hainitpa hinashtuyakaiikana akamkinikom taaiskitki hinashtamtainaiikana uyikalaki kikomainishna wakash hinashaniaiikana, uyikalaph titoshiph wakas hinashaniaiikana.

$2+\mathrm{Ki}$ mat uyikashlipkinih Syriapkinih pauimsiashama ipna kut; kunki pa winahpaikoshama uyikala kikomainin, uyika!a titohohshin, uyikala kikamimlainin, uyikala didevilhin, uyikala kikishinin, uyikala lilkupshimainin; kaua uyikalana wakas hinashwihania.

25 Kaua ilahniwam titokanı ipna patuanihnikshana Galileepkinih, Decapolispkinih, 
Jerusalemkinih, Judeapkinih wah Jordan nakaikinikaikinih.

\section{WANAHNA V.}

T A kaua ilahnina hinashahna kaua kuh1 shiph hipalatia kaua hiuhshalika, kaua ipnim Disciples ipna patuihsana:

2 Kaua hinashna immuna

3 Yiyaunin hiutsatanih ka imma immamahuitwitanih wakaswith: immain autsatanih aishniwash Akamkinikaih.

4 Yiyaunin hiutsatanih ka immam immamathautanih: ima shapaliloinin autsatatashi.

5 Yiyaunin ka imma talhtalh hiutsatanih: imman watash autsatatashi.

6 Yiyaunin hiutsatanih ka imma haiyaktanih hikayautanih tsaph: imma kos hitkailaktatashi.

7 Yiyaunin hiutsatanih ka immam paiyautanih imma paktatashi yiyauna.

8 Yiyaunin hiutsatanih ka immam kaihkaih timina autsatanih: immam paktatshi Godna.

9 Yiyaunin hiutsatanih ka immam patatktanih; immuna puniktatashi Godnim mamaias.

10 Yiyaunin hiutsatanih ka ima toptakin hiutsatanih tsawasatki immam aishniwash autsatanih Akamkinikaih.

11 Kapain imuna ath hitoptakitatashi, ath hiwaptakitatshi, ath hipshitimtatashi, uyika- 
laph, imamnihki mishamitki In wasatkisim: kaua ima ath yiyaunin hiutsatatashi.

12 Kava ath paishnu ath paliloinikiu; himakas init imam ath washi aishnawashpa; matu ku kush pauyaoptakashana imam nokunmana haimait:inpuna.

13 Ima ath washi katuanash watashain: tsaluin katuana hipaamnah, kaua ituki hipakatuatoktah? kaua kunih watu ituain kala wakainash titokanm watiksatash.

14 Ima ath washi sapalakauiotash watashaian. Yoh ka hiwash tauyanikash kuhshpa watu manmaiih shapalukash hiwash.

15 Ka kaua patuapiptanih sapalakauitashna kaua watu hikaiki shaukolikasatash hiwash, kala hipalikasitanih ipalikasatashpa; kunki hinatslakauiosha uyikalona ka kalo initpa haastanih.

16 Kauo ima asapalakauioth titokana, kaua ath hipahnanyukum imuna tahs wiakut kunki imam Totaph aishniwashaph, potasktatashi.

17 Wat mat panakukum Ina, kuh tamaluit hukaitama hu ituna haimaitsinpuna: watu wakuitama, kala tsatama.

18 Ikuin ath hisa ki watash wah akamkinikai laamnash hiwash, matu watu manmaiih kashinash tmaluitkinih naksnih yot, hu itu samuh, laam hiwash tsanash.

19 Ka ipnim paukaitatasha naksna kuskusanihna tamaluitkinih, kaua uikinih pasha- 
paukaitatasha titokana, ipna puniktatashi kuskusanik Aishniwashnim inakniktph: kaua taklai ka ipnim hitsatatasha kaua uikinih titokana pashapatsatatasha, ipnatita puniktatashi takash aishniwashnim inakniktph.

20 Ath hisa kapam watu ath ima akatosktatashi Titimanawatona wah Phariseena tsaki kaua watu manmaiih atk astatashinki aishniwashnim inakanikitph.

21 Wako ath sukuanaisha imamanokunmana wiatsekin, hinanihna wat mat opsiaunu ka ipnim popsiaunu ipisim shapatkukash hashwalauitki hiutsayu.

22 Matu ath hisa ka ipnim lilkuh pahahsamnotatasha lautuana ipi shapatkukash hashwalauitki hiutsayu: Ka ipnim Lautuana patanuetatasha, sili awash, ipi shapatkukash tamaluitki: Ka ipi lautua hitalaulakitatasha, palepale awash ipi wakainash alaph animkinikaiph.

23 Kunki kam kaua im aunahpaikoyu talamionashna talamionwashpa, kaua pilh a kiyu kuh kush in lautuam hitimipniosham,

24 Kauo talamionashna anwihnanu talamionwashpa kaua akiuyu; im lautuanankos ahimiyunu kaua atalamionu.

$25 \mathrm{Kam}$ kaua imana hinahnatatasha hashwalauitph kos wislampa; hamtis a.wasatitah im iniatona kainam imana iniatom hinahpaiktanu haswalauitph, kainam hashwalauinm imana hiniku shaktishph initph. 
26 Ikuin ath hisa watu manmaiih atoktatasham kunih wasatiaiph; inakih kuskusaniph.

27 Wako ath sukuanaisha imamanokunmana wiatsekin, hinanihna wat mat pahweyu: 28 Matu In ath hisa ka ipi hisilauwauluksha aiatona, wako pahweya hiwitsas timnaki.

$29 \mathrm{Kam}$ kaua imim wapsuhpkinikainim silunm imana hishapakapshishwiyu, kaua A imankashupanyu kaua a imaukainanyu: matu kum im tahs naksni patish wakainah; matu watu tahs wakainash wilwilp silakt animkinikaih, alaph.

$30 \mathrm{Kam}$ imim wapsuhpkinikainim ipshushnim imana hishapakapshishwiyu, kaua a imshakiuaukyu kaua A imaukainanyu: matu kum im tahs naksni patish wakainash, matu watu tahs wakainash wilwilpsilakt animkinikaih, alaph.

31. Hinanihna ka ipa iwapna hiuyuinupaswiyu ipnim ush niwihnaitash iwapna puyuinin timash.

32 Matu In ath hisa ka ipi iwapna pahwiaina hiuyuinu ipi iwapna hishapapahweyu ; kaua taklai ka ipnim Kunia tinunimna panpu ipi pahweya, wako hiutsayu.

33 Wako ath sukuanaishi hanaka imamanokunmana wiatsekin, hinanihna wat mat, ashaku, kam kaua tsehnu Lordiph yoh A tsekin tuihnu kunku : 
34 Matu In ath hisa wat mat imanahkiunu hu ituki akamkinikaiki; kala ush Godnim wahshilikasatash.

$35 \mathrm{Hu}$ ituki watashki; kala ush ipnim watikash: hu ituki Jerusalemki; kala ush himakasnihnim miohatom tauyanikash.

36 Wat mat im hushushki imanahkiuyu, watu imim kapshiki naks huku imaniaitash haihai hu ma simuhsimuh.

37 Kalo ath hitatashi A, A; Watu, Watu: kapam kunia ath apawaunu yoh ath kapshishwitki kutatashi.

$38 \mathrm{~W}$ ako ath sukuanaisha tsekin, hinanihna siluki waptaklaikash siluph, wah titki titph. 39 Matu In ath hisa wat mat imoptakiyu: kapam, wapsuhpkinikai mastai hipaptanyu, kaua ath sahiah kinikai aunikoya.

40 Kam naksnim hamanm hinpanyu shamah tamaluitki sauin wat mat yauiyu siskan.

41 Ka nakski ipnashapatuihnu naksiph mailiph kau uikinih lapitiph mailiph $\mathrm{A}$ atuihnu.

42 Kam ipnim imana hinahmunu waya $\mathbf{A}$ auniyu, kam ipnim hiwamshitapaswayu wat mat ipna akailonapiku.

43 Wako atk apamsianyu tsekin hinanihna, im lautuanasim ahatauyu, matu im tuelkana A simhnu.

44 Matu In ath hisa im tulkana apahatauyu, apayiyaunu kapam immam hitimnopsiauta- 
nih, apashapatahswiyu kapam immam hahehsamnotanih, apoyiyaukoyu kapam immam hitalilkuktanih, kapam immam hitoptakatanih.

45 Kuki ima ath witsatatashi im Totam mamaias aishniwashpa: matu kupam ipnim hiuyashapatilahtotatum kakapshishinahna, titahshinahna, hiuyashapokiyotutum tsitsanishna, wah tsitsashimaina.

46 Kapam imam imuna ahatauitanih Kuniasim atk apahatauitah wak ituna apakinah? Imat watu Kush hikutanih hinpaiikawat?

47 Tsalaui im lautuanasim atk apasuyasaiyu, wak ishimana Kaua atk apakatosku? imat watu Kush hinpaiikawat hikutanih ?

48 Waya imamashapatsath Ka Kush imam Tot Aishniwashpa Ipnashapatsasa.

\section{WANAHNA VI.}

TAHAINAPAM washina wat mat ima11 mashapaki apayiyaunu: Kainapam watu ituna apahnu imam Totaph Aishniwashpa.

2 Kunki Kapam Kaua ima apayiyaunu, wat mat apashapunminkosiyu, Ka kush Kolwaktipas hikutanih ipnoyiyaukinwashpa hainitpa, wak iskitpa imamashaposhaiktash titokaph. Ikuin ath hisa watu hikolwaktanih.

$3 \mathrm{Kam}$ kaua ayiyaunaiiku wat mat wapsuhpkinikaina ipshushna wiakut ashapasukuananyu sahiahpkinihkaiph ipsusph : 
4 Kunki ayiyaunaiiktah peleispa: Kunki im Totam, Kam ipnim peleispkinih haktatum, ipnim a hiwasatiukum, Kaiih.

5 Wat mat imoyiyauku Ka kush Kikolwakin hikutanih kala imamashapahnash immamoyiyauktipashwitanih ipnoyiyaukinwashpa, hainitpa wah piksaniwashpa iskitpa, Ikuin ath hisa watu hikolwaktanih.

6 Kam Kaua imoyiyauku Kaua A asu im initph, Kaua A imokalku Kaua im Totaph imoyiyauku, Ka ipi peleispa hiwam, Kaua im Totam peleispkinih haktatum, ipnim a hiwasatiukum Kaiih.

7 Kapam Kaua ath imamoyiyauku wat mat apolamhlamhnu, Ka Kush Gentilpum hiuyakutanih, immamanaktanih nuna ilahniph tsekiph hinatsmitsitatasham.

8 Wat mat Kunmana apatuihnu: Kos ath tsehnaipa, wako imam Totam hisukuanaitatum imuna hiuituit.

9 Kunki kush atk imamoyiyauku Nunim Pisht Aishniwashpa imim wanikt hautnin Kam watu.

10 Imim miohatoit ki anashapautsasham, Imim Kutki anashapautsam uyikashliph Ka Kush aishniwashpa, hikutanih.

11 Taks lahaipa hipt natsnim taksain.

12 Nuna wasatiai nashwaunim Ka Kush nun titokana wasatiai awaunaitanih.

13 Wat mat anashtahinawiyukum nuna, 
matu taklai nuna shapakapshishwiatupkinih natsnahwuinukum: Imim awam inakanikt, imim awam Kapskapsnawit imim awam siskeiwit Kunku. Amen.

14 Kapam ima ath titokana Kapshishwit apawaunanyu; Kushtit Kaua imam Totam aishniwashpa, imuna Kapshishwit ath waunanyukum.

15 Matu Kapam watu ima titokana Kapshishwit apawaunanyu Kushtit imam Totam watu ath hiwaunanyukum imuna Kapshish wit.

16 Kaua hanaka Kapam kaua ima atk imamauyashapahyahnu watmat kol wakishna apatuihnu, Kala immokiasktanih nahshap mastai immamaniaitanih, Kunki titokaph immamashapahnahs imamashapahyaktanih. Ikuin ath hisa watu hikolwaktanih.

17 Kam Kaua im wiahyahnu imamatuaiku A wak ipshimataiu A.

18 Kunki watu imamashapahṇash titokaph a wiahyahnu, taklai im Totaph peleisph awiahyahnu: Kunki im Totam, Ka ipi peleispkinih haktatum, Kunim a hiwasatiukum Kaiih.

19 Wat mat imamanikanyu Kuishwit kina watashpa, atka Kina hiutsatatu kalkal wah puhpuhwit, Kina pahweya hinkaskilkaiikshi, hipahweaiikshi.

20 Matu taklai Kusihwit atk imamanikanyu aishniwashpaisim atka Kuna saiau hi- 
wash Kalkal, saiau puhpuhwit, watu Kuna pahweya hinkaskilkaiiksha, hipahweaiiksha.

21 Kapam minah Kuishwit panikitah, Kunapkitit timina ath pautsayu.

22 Silunm hiwash sapalakauiotash silakana: Kunki tsalui imim silu Kaihkaih awitsatah kaua imim silakt lakauitpaisin awitsatah.

23 Matu kaua taklai tsalui imim silu shilmakshin awitsatah, Kaua imim silakt shaktitpaisim awitsatah, Kunki tsalui imim lakauit tahash kutah, Kaua komais shaktin awitsatah.

24 Watu manmaiih lapuana inakanikawatona tuihnash titokanm hiwash: Atka naksna pahtauitah, Kaua naksna pasimhnah; hu ma naksna patuihnah, Kaua naksna patalhnapiktah: Kunki watu manmaiih ath washi tuihnash Godna wah Mamona. (Kuishwina.)

$25 \mathrm{Kunki}$ ath hisa wat mat imam wakaswit imamakesknanyu hu ituki hipitki hu ituki imamakutki: Kaua hanaka watu mat imam silakt imamakesknanyu imamauyashapashamkitki: Mish watu wakaswinm panoktiaisha hipina? Mish watu silakanm panoktiaisha shamahna?

26 Waya akith waiyutalikna akamkinikaih; watu maua hitamaniktanih, watu maua hituptanih watu maua hinakainaktanih inikash; sauin imam Totam aishniwashpa hinashapaptatum. Mish im watu aunoktiaishi Kunmana. 
27 Ishima kinama naks ipnim timiuki ipnashapapiimnash naks kaptkapt uikinih ?

28 Ituain imamakesknaitanih shamahki? waya timipnith lonasna tahpamph, manmaiih hiuyapiimsi? saiau sapalihlikt awusatanih, saiau watukit awusatanih :

29 Sauin ath hisa Solomon watu naksna kinama pawauna siskeiswitki.

30 Kala Godnim wilatina pauyopaliktatum, matu taksainsim hiwushi, watishkinain hiwusha tulailakash tamashph. Mish watu imunanka hishapashamkitatasham? Atapam imam kuskus mitskuanakt waishi.

31 Kunki wat mat imamakesknu, wat mat panaku itunanm aptatashi? itunph kanm numakutatashi? itu nanm shamkitataishi?

32 (Kala kusnasim papawiaiiktanih Gentilpum :) matu imam Totam aishniwashph ath hisukuanaitatum imam huituitkinaph uyikalaph.

33 Waya Godnan kos aishniwash anpukinyanith wah ipna tsanash, kauana ath hiniyukum ki uyikala.

34 Kunki wat mat watishkinain imamaluhluhnanyu: kos watishnimsim ipnalahluhnantash hiwash kunainikain ipnimnikain. Taksama kapshishwit taksain hiutsatatatu.

\section{WANAHNA VII.}

WAT mat apatimnalawakoyu, kainapam imunanka hipatimnalawakoyu. 
2 Kapam kunki timnalawakotki apatimnalawakoyu kunkita ath imunanka hipatimnalawakoyukum; kapam kunki shapinauitaski apashapinauianyu, Kunkitita shapinauitaski ath imunanka hishapinauiyanyukum.

3 Ituain ahnaisha imskapnasim siluph kapshin: Matu watu A imatimipniaisha silupa awash wiwisa.

$4 \mathrm{~W}$ ah manmaiih im hitatasha aska Kama kapsin imakahtanish imim silupkinih, matu awah, imim silupa awash wiwisa.

5 Kolwakin imkos imanakahtanih wiwisa imniphkinih siluphkinih, kaua kam lauit hakiki imskapna kapsin A aunakahtanyu silupkinih.

6 Wat mat hautpama sikamkalna apakuya$\mathrm{ku}$; wat mat imam patopato hokhoknim anoktkinikai apawitqaitanyu, kainapam hipatowatiksanyu, kainapam imunanka hipayumtipu.

.7 Aunahmuth kaua ath hiniyukum; apawith kaua taks ath apayahnu; pishkish aptanith kaua ath taks hitamahalpanyukum.

$8 \mathrm{Ka}$ ipnim panahmutatu ipna panitatum; Ka ipnirn pawiaiiktatu, ipnim payaktatu; Ka ipnim pishkish paptataitu ipna patamahalpaitatasham.

$9 \mathrm{Hu} \mathrm{ku}$ naks imamkinih hama hiwatah, ku miahspim panahmunah hipitph, kaua mish taklai pishwa panitah? 
$10 \mathrm{Hu}$ ku suyamph panahmunah mish kaua taklai tukumutalikin panitah?

11 Inaki ath kakapshish washi sauin imam mamaiasna patu titahs atk aunitanih imam timiuki, mish watu imam Totam aishniwasph uikinih mitsitash, Kunki patu titahsna hinatsnitatasham inahmuph?

12 Kapam kush ima imamanaktanih, nash mish kush titokanm hipanashkotamh nuna uyikalaph, waya imankos kush auyakuth titokana uyikalaph, kapam kush imamanaktanih; yoh hiwash tamaluit wah haimatsinpun.

13 Yohsim tukuh pishkish hiwam kunapkisim astath; kala himakes pishkish hiwam Kaih ishkit hiwam wakainiwashph, Kunapisim ilahni hiwiwahyasinki.

14 Tukuh pishkish hiwam kalalish ishkit hiwam wakeswitph kunki milasnim kunia paiyaktanih.

15 Aishauth mimishamina haimatsnipuna, Kapam kunmam hipainoyiktanih, shipnim makaski ipnathtashamkin, matu awiushi himin imit kasiliktipas :

16 Ath auyashuktatashi immuna wiakutpa. Mish maua titokanm hitmanitanih shishnim sauaipa grapena, hu ma itu figna titohshauwaipa?

17 Kunki titahspaisim taulikitpa auyapiimtatu titahssim timanit; Kaua taklai kakapsh- 
ishpa taulikitpa auyapiimtatu kakapshishnim timanit.

18 Watu minmaiih tahsnim taulikinm shapautsatash kakapshish timanit; Kaua taklai watu manmaiih kapshishnim taulikinm shapautsatash titahs timanit.

$19 \mathrm{Ka}$ kalanim taulikinm saiau hishapautsatatu titahs timanit, kunia pokiuktanih shapalunash.

20 Kunki timanitpa atk apashuku.

$21 \mathrm{Ka}$ ima hitanih Inimph LORD, LORD, watu uyikalo atkautatashinki aishniwashph Akamkinikaih. Ka ipisim Na Totam Aishniwashph wiakutki hiuyakutatu, ipnimsim atkaunash.

22 Yoh ka naks lahain hiwaki inaki kuna ilahniwa hitatashinm Inimph, LORD, LORD, mishah watu imim wanikitki nu himatsinpanihna? mishah watu imim wanikitki pashapatanihna didevilna? mishah watu imim wanikitki paminmaiih hititwatityayanauwianihna?

23 Kaua kunmana In inatsikuitimtatasha, watu maua ath In sukuana shapakapshishwiat ath washi, niwihnamth.

$24 \mathrm{Ka}$ ipnim Ina tsekin himsianyukum kaua kunkism hiuyakutatasha, kunia In ka shapasas nakski wapsuhki hamaki ka ipi hania init pishwepa :

25 Inaki pokioanima, inaki pahatianoanima, 
inaki patkaikotamaunima sauin watu hitkaskilka: Atka pishwepa haniin hiwaka.

26 Kaua taklai ka ipnim Ina tsekin himsiaisha matu watu kunki hiuyakusha, kunki pashapasatatasha nakski atalpishki hamaki ka ipi hania init maiahpa :

27 Kaua pokioanima, kaua patalanoanima, kaua pahatianoanima, kaua patkaikotamuanima, ki mat hitkaskilka, atka maiahpa haniin hiwaka: Kunki himakes waukainin hiutsaya. $28 \mathrm{Ka}$ kaua Jesusnim uyikalaki tseki hinashwihinakia kaua uyikalo titokanm hipasiwaina ipna tuyakitph.

29 Atka titwatityayapama hinashtuyakima, watu ka kush titimanawatonm patuyakanihna.

\section{WANAHNA VIII.}

T A kaua hitamika kuhshpkinih kaua ilah11 ninm pauwatasnim patuihsina ipna.

$2 \mathrm{Ki}$ mat naksnim Leprosnim papainoya ipna, patqoikoklikoya, pana Lord imim timiuki kum kaihkaih shapautsatamh.

3 Kaua Jesusnim patkapalikasaya, pana inim timiuki, kaihkaih witsaih : Ki mat kunai nih kaihkaih hiutsaya Leproskinih.

4 Kaua Jesusnim pana ipna; Mitsim, wat mat ishina aunu; akiuyu Talamionawatoph imashapaktah, Ka yoh Moses hinashimtaka, kunmaiih atalamionu imashapasukuanash titokaph. 
5 Ka kaua Jesus Capernaumpa hipaina, ki mat naks Centurion papainoya ipna; ipnoyiyauktama.

6 Pana, Lord, inim waiimtas hiisa initpa, likupshimai, komais shapatelpin wash.

7. Kaua Jesusnim pana kos tuiksa wakas A haniaitasa.

$8 \mathrm{Ki}$ mat Centurion pamstakanpa, pana Lord, inalapkiyaunin wash watu asaitash awash ina inith, kala imim tsekisim kaua inim waiimtas wakas witsatash.

9 Kala Tamaluitki wash; wak in uikinih anatsnakniksha piwapsiaunatona, In naksna atanuetatu kuih, Kaua hikutatu naksna autatu Kium, kaua hikiutatum, kau waiimtasna autatu Ki hanianim, kaua haniaitatu.

$10 \mathrm{Ka}$ kaua Jesusnim pamsia kaua hiswaina, kaua hinashna ka immam patuihsana Ikuin ath hisa watu mina kashlana mitskuanakna auyaksaka, inaki Israelpa.

11 Ath hisa kos ilahniwa piamhtatashi tinahtitkinikaikinih, wah tilailakitkinikai kinih, Kaua Abrahamnin wah Isaacnin, wah Jacobnin hitamayahtuatatashinki aishniwashpa Akamkinikaih :

12 Matu inakanikinwashpama mamaiasna pashuyalahtatasha shaktitamaunishph, kaua kuna hiwitatashi hititkanptatashi.

13 Kaua Jesusnim pana Centuriona (putaptit inakanikawatona), siklin Kam kashl imim 
mitskuanakt wash, kashtit awitsatatasha. Ki mat kunmainih wakas waiimtas autsaya.

$14 \mathrm{Ka}$ kaua Peterna initph paasoya Jesusnim, Ki mat ipna siwako pahnanya hiisa ilaikaiiki hikomaisana.

15 Kaua ipna ipshush popasanya, $\mathrm{Ki}$ mat hilaiknim panwihna: Kaua ata hushakaika kaua immuna hinashapalihnakanya.

$16 \mathrm{Ka}$ kaua hikulawitna kaua ipna ilahni panahpaikoshina Didevilitin, Kaua ipnim tseki spirits hinashwishapataya, uyikalona kikomainishna wakas hinashwihania:

17 Kunki Esaiasnim, imatsinpunm tsekin atsanima, yoh Ka hitsehna, ipinih Kia nuna ilatuwit hinpanima, wah nuna komain Kia hinakanikanya.

$18 \mathrm{Ka}$ kaua Jesus hinashahna ilahni pauwatashna pelkai patamalkalika Kaua hinashna Ka apawaiiks.

19 Kaua naksnim timanawatom papainoya pana Miohat A tuanihniktatasha Kunku.

20 Kaua pana ipna Jesusnim inaki tilipanm hiumnit awiushi inaki waiyutalihnim awiushi shiks; matu hamanm Miahspim watu mina wautalikash ush.

21 Kaua hanaka naksnim ipnim Disciplepim pana Ipna Lord kuh In pisht tamiktanah.

$22 \mathrm{Ki}$ mat Jesusnim pana ipna, Ina tuihnim, kos ka titinuhnishnim titinuhnishna patamikshanah: 
$23 \mathrm{Ka}$ kaua liash hipawasakika, kaua inakanikitpim ipna patuiksana.

$24 \mathrm{Ki}$ mat kapish hinashatianoma iwatampa, liash hinashtamalapslapsnanima, hauwinim; matu ipi hipanimshana.

25 Kaua ipnim inakanikitpim pashapohna pasana Lord nashyiyaunim: Kia takoshi.

26 Kaua hinashna, ituain sikausi? atapam kutskuts mitskuanakt washi, kaua hilaushakaika, kaua hatiana wak iwatamna hinashtatka. Ki mat kunmainih lamlamat hiwaitalkima.

27 Kaua hahamnim patsiwaina pishapnia, manama, minama ki hama hiwash ata hatianm wah iwatamnim pamsitatu ipna?

$28 \mathrm{Ka}$ kaua hipatkekika Gadarapa watashpa Ki mat lapiuam didevilhishnim pawaukunia ipna, shawaipkinih; sisikauishnim: Watu manama kunapi hikiaiikanihna titokanm.

$29 \mathrm{Ki}$ mat yokamam patyohnokinishana; pasana Jesus Godnim Miahs mïshah imana tuihnash washi? Ituph kuma, mat nashapaqoimtama? Matu kos watu tsasi.

30 Matu hiwaka waiat naks asa hokhok ilahni, hiwaukupshina.

31 Kaua didevilm pashapnia ipna, pasana tsalui nuna A nasuyalahtukum kuh hokhokph paastah.

32 Kaua hinashna, A, kutatku. Ka kaua 
hipaatkika Kaua hokhokph hipaaskika, ki mat laam naks asa uyikala hokhok tushkinih hitalwaluya iwatampa, laam hitkoya kushpa.

33 Kunki walahnawatom hipanahwulakaika tauyanikashph panahwilapaikoshana uyikalapama patamapaikoshana: Wah lapuaki ka imam didevil itin hiusheka.

34 Kaua laam tauyanikashkinih Jesusna patkakiokinishana: Ka kaua ipna paksana kaua pasana, nashniwihnamh hamtis num watashkinih.

\section{WANAHNA IX.}

T AUA liashph hiwaiiktoka, ipnim tauya11 nikashpa.

$2 \mathrm{Ki}$ mat lukupshimai, hama panahpaikoshana, pinmikashina; ka kaua Jesusnim hinashahnanya imınuna mitskaunakt, lukupshimaina kaua pana Nisu waya aishin, wako A waunin wash mitsitpashwit.

$3 \mathrm{Ki}$ mat tatoshmam titimanawatom hitimnatseksina ; manama panahmishamsa.

4 Matu Jesus hinashsukuananya immuna timnatsekin, kunki hinashna ituain kapshishwitki timnanakshi?

5 Ma hi alauis kuh In hinah kama waunanish mitsitpashwit; hu kuh hinah, wishakaih, wihna.

6 Sauin ath shapasukuasa, hamanm Miahspim ush waunash mitsitpashwina watashpa, 
kunki lukupshimaina pana, wishakaih, pinmikash iniph, kaua siklin im initph.

7 Ki mat hushakaika ipnim inithpa hipaitoka.

$8 \mathrm{Ka}$ kaua piamhnishnim paksina, kaua hipasiwaina, Godna potahskshana, ipnim initki, yoh ka titokana kush kapishin hinatsnia.

9 Kaua kunih hikuya. Kaua ata naksna hamana pahna unikshana Matthew inpukininwashpa hiuhshalika: Kaua pana, tuihnim. Kaua hushakaika, kaua patuihna.

10 Kaua Jesus Discipleshina initpa hipshina; Ki mat ilahninm silpsilp hinpaiikawatom. wah mimitsitpashnim patamayakaktuasana.

11 Ka kaua Phariseepum paksana, kaua Ipna Disciples pashapniaishana, manama imam Takash Ipnashapakuautuasa, mimitsitpashki wah Custom hinpaiikawatoki?

$12 \mathrm{Ka}$ kaua Jesusnim hinatsmitsia, kaua hinashna, watu maua wiwakesnim imamashapakiutash saikiptawatoph; Kala kikomainishnimsim.

13 Kunki kutath waya sukuath naksna tsekna, yiyauna In ashaihtatu wah watu talamionashna. Watu mish tsitsanishna amutama, kala mimitsitpashana ashapamsukuanaktama.

14 Kaua Johnm Disciplespim papainoya 
kaua pana ipna, manama watu imim Disciples Hiuyahyahtanih matu nun wiahyahtanih wah Phariseepu.

15 Kaua Jesus hinashna, mish tamasam mamaiaspim initpa shapakomnash tamashina? Kos tamas hinatsinpaitataisha, kaua hiuyahyahtatashi.

16 Watu maua titokan kimtiki simkakna tiktamailakash kaina tiktamailahnim pankaukainu simkakana kaua uikinih tatnin himakas hiutsayu.

17 Kushtit watu titokanm kimti sisyukin hiuyayakakasatanih wiwakemaph itatpashph, kaina tuhs itatpash hikiu; sisyukin laam haatu, haua itatpash hilaamnu. Matu pauyayakakasatanih kimti sisyukin kikimtiph itatpashph, kaua uilapti wakas witsatatu.

18 Kos hinashtuyakshana immuna; Ki mat naksnim miohatom papainoya, patqoikoklikoya ipna, pana inim timaiimias wako tinuhnin hiwash, kum Im tuihnamh; Kum opasatah ipna, kaua wakas witsatah.

19 Kaua Jesus hiushakaika, kaua patuihna ipna inakanikthina:

$20 \mathrm{Ki}$ mat naksnim aiatom amtinuhnishnim, wako putama inwim wah lapa inwim kashl amtinuhsana, ipnim halakinih shamah talampa panpaituihna.

21 Ipinih timnatsehna; kuh In shamahnasim opasatah kuh wakas witsatah. 
22 Kaua Jesusnim pakailaunoya, ka kaua pahna ipna, kaua hihina, Nisu, aishin waya; Im mitsikuanakitki wakas, A witsas. Kauo kunih yokopi aiat wakas hiutsaya.

$23 \mathrm{Ka}$ kaua Jesus haasa miohatom initpa; Ki mat kaua hinashana shapunmiatona, wah ilwahwahnatona.

24 Kaua hinashna witsapikimth; kala watu tinuhnin Ki timai hiwash; Kala hipnimsha: Kaua immam pasapinmishana ipna.

25 Matu ka kaua pashapatnakia uyikalana; kaua paasoya, ipna ipshush panpanya, Ki mat yokopi timai hiushakaika.

26 Kaua kunkinih siswaish hikiaiika uyikashliph wiwatasph.

27 Ka kaua kunih hikuya Jesus ki mat lapuam hailatamam pamunatuihsina, ipna pasina, Davidnim Miahs nashyiyaunim.

$28 \mathrm{Ka}$ kaua initpa haaskika, Kaua hailatamam pasoatuihna; Kaua Jesus hinashna mish ima ath mitskuanakshinm; Ka inim kapshiki wash kus kutash? Kaua pasina, A, num Takash.

29 Kaua silu hinashwitkopsakanya, hinashna kapam kashl imam mitskuanakt washi; kashtita ath witsatatashi.

$30 \mathrm{Ki}$ mat kaua hipasaiohoya: Kaua Jesus hinashtatka, hinashna wat mat titokaph imamashapasukuanu.

31 Sauin ima hipakuya, ipnasiswais kut 
panahtamtainaiikaishana, uyikashliph wiwatasph.

$32 \mathrm{Ka}$ kaua hipaatoka kaua ata panahpaikoshana naks hama tsekshimai, Devilitin.

33 Ka kaua ipna Devil pashapatanya, kaua ata tsehshimai hitsehna; Kaua pauwatasnim pasiwaisana, pisina kat watu maua kush Israelpa aksina.

34 Matu Phariseepum pisina, Kala Didevilm miohatoki Didevilna hinashapatatu.

35 Kaua Jesus hinashkiaiikoya uyikalapa titauyanikashpa ; uyikalapa wiwatashpa, hinashtuyakaiika ; ipnoyiyaukinwashpa hainitpa, aishniwashpim taaiskitki hinashtaaiskaiika; uyikalona kikomainishna wah uyikalona hailana wakas hinashaniaiika.

$36 \mathrm{Ka}$ kaua piamhnishna hinashahna, kaua komki hinashtimnayiyauna; uyikalo tamaushilikin hiwiushina Ka kush ship pipuhnin walaknawatanotpa.

37 Kunki ipnim inaknikt hihina, ikuin atu wako ilahni lilaken hiwash, matu milastamaun titupnawat.

38 Lilaken Inakanikawatona, waya, oyiyaukoth ka titupnawat hinashapakium ipnim lilakeshph.

\section{WANAHNA $\mathrm{X}$.}

K A kaua ipnim Disciplesna putimwana 11 wah lapuwana hinashmuna; kaua hi- 
natsnia kapshin Didevilain shapataiikash; wah kikomainishain wah titoshinain shapokaswitash.

2 Shapawihnanishnim (Disciples) putimwam wah lapuwam wanikit aushina kush. Naksip Simon ka kunia punika Peter, wah ipnim askap, Andrew: Kaua Jaines, Zebedeenm, miahs, wak ipnim askap John;

3 Philip,wah Bartholomew; Thomas, wah Matthew hinpaiikawat; James, Alpheusnim miahs; wah Lebbeus, ka kunia punikshina Thadeus;

4 Simon, Cananpu; wah Judas Iscariot, ka kunim patamapaika ipna.

5 Kinmana putimwana wah lapuwana hinashwishapawihna Jesusnim; hinashtuyakatuihna, hinashna wat mat Gentilepuna iskit apaasaiikanyu wat mat Samariapuna naks tauyanikash apaasanyu.

6 Kala Israelpum initkinih, pelenishna ship. na awituikith.

7 Atk apatamtainaiiku, Atk apanaiiku aishniwashnim inakanikitpim wako hikakamnisham.

8 Kikomainishna wakas atk apaniaiiku: Lileprosna atk apawisapakaiku : Titinuhnishna atk apawishapautalahtaiiku; Didevilna atk apashapataiiku. Wasatiai ath washi init; kushtit lilku atk apania.

9 Wat mat imamasauyawanu itatpashpa, Goldki, Silverki, Brasski : 
10 Wat mat imamasauyawanu ishapski, hu ma lapitiki shamahki, hu ma tukashki, atka sapalihnikawat ipnim lukswitki ipnashapapaiikash hiwash.

11 Kapam kaua mina nakspa tauyanikashpa himakaspa, hu ma kutskutspa tauyanikashpa atk apaasoyu, kaua atk apawishapniu ma hiwash payaunipas kaua kunainih atk apatauyaniku, kapam kaua kos ath apawihnanu.

12 Kapam kaua apauyasoyu hainitpa, kaua atk apahimiunu.

13 Tsalui init himiunipas hiwaku kaua atk apahimiunu, Tsalui himuishimai hiwaku, kaua atk himiu panpatuihnu.

14 Kapam ipnim watu ath himiunukum, wah watu ath himsianyukum tsekin, kapam kaua kunmana initkinih atk apatapiku, hu ma tauyanikashpkinih, kaua atk apatulahimhnihnanyu ahwapama ith.

15 Ikuin ath hisa kanm piamtatashinki shapatkukinwashph kaua yokopnim tauyanikashnim Sodomna wah Gomorahna pawautatashinki wakaiki.

16 Kushka shipna ath shapawihnasa himishph, kunki ka kush tukumutalikin kush ath popsukuiwiaiiku; matu ka kush koinu kush ath paamqamnawiaiiku.

17 Titokana atk apashiaunaiiku, Kala atk hiuyanahnatatashi hashwalauitph, ath hiuyawawiatatashi ipnoyiyaukinwashpa hainitpa. 
18 Mimiohatpa, wah titamalukawatopa ath hiuyanahnatatashi. Inwasatki tamapaikash imamain wah Gentilepuain hiutsatatasha.

19 Kapain Kaua imuna ath hipanahnanyu wat mat Kaua panaku, nas manmaiih tsektatashi, Kos ath Kunainih hishapatsehnukum.

20 Watu imamnih ath hiuyatsektanih Kala imam Totamsim Spiritkisim ath hiuyashapatsektatum.

21 Kos piapim shapopsiautatasha askap, hu ma askapim; Kushtit miahs; hishapopsiautatasha kaua taklai marnaiaspim pothohalikotatashi pisht, pika kaua hishapopsiautatashi.

22 Inm wanikitwasatki uyikalom hahehsamnotatashi imuna, matu Ka ipisim ipnauyashapahtuktatasha lamtaiph kaauph, ipisim wakas hiutsayu.

23 Kapam kaua imuna hiwaptakatatashi nakspa tauyanikashpa, Kaua ath kunih apaulalihnapiku naksiph; Ikuin ath hisa, watu laam Israelpum titauyanikashpa atk akiaiikotatashi, Kaua wako hinashpainotatasham hamanm Miahs.

24 Wat maua inakanikitpim inakanikawatona hiwautatu;; Kushtit watu maua hashwalaianm pisht hiwautatu.

25 Tsalui inakanikitpim inakanikawatona kunapitita patuiktanih, kaua watsu. Wako inina inakanikawatona punikshi Beelzebub 
mish watu uikinih ipna mamaias kushtit punikaitataishi.

26 Wat mat kunmana apaskaunu. Uyikala, Ka kala ipnashtakin hiwiushi, laam. shapahnash hiutsayu. Uyikala Ka kala shapalukin hiwiushi, laam shapasukuanash hiutsayu.

$27 \mathrm{Ki}$ kapama In ath tanuesha shaktitpa, Kaua kunki tseki ath auyatamtainaiiktatashi hilakauishpa; Kapam kunia atk amsitanih sausauipa kaua kunia tsehna atk apatamapaikaiiku ininm sukuipkinih.

28 Wat mat Kunama atk apaskaunu Ka imam silakanasim wapsiaunash awiushi matu watu manmaiih wakaswina wapsiaunash awiushi. Ipnasim atk apaskaunu, Ka ipi hiwam wakainash silakana wakaswithina, alaph, Animkinikaih.

29 Atka lapit payupayu pitamaiat hiwash naksniki Farthingki; sauin watu naks kunama, takauitash watashph, imam Totam sukuanai.

30 Uyikala imam hushuspama huku, wako hitămanin hiwash.

31 Kunki wat mat imamanahsikaunu; Ilahnina payupayuna atk akatoskshi.

32 Kunki ka ipnim Ina hilapsqiaiyu titokaph, Kunia In taklai atamapaiktatasha NaTotamph Aishniwashph.

33 Kaua taklai Ka ipnim ina watu himsi- 
lapayunu titokaph takalai Kunia In watu amsilapayunu NaTotamph aishniwashph.

34 Wat mat ima apanakukum tatkash hishapautsatama Kina watshpa, watu tatkash In shapautsatama, Kala wals ashapautsaiiktama.

35 Kala hamana In shapatoptakitama pishitph, wah otwaina pikaph, wah aiatona pinahshiph.

36 Hamanm ipnim initkinih tuelkauyuin autsayu.

37 Ka ipnim pishitki hu ma pikaki Ina hishapakatosksha hatuitki, ipna Inim watu hatauitash. Ka ipnim haswalki hu ma pitiniki Ina hishapakatosksha hatauitki ipna Inim watu hatauitash wash.

$38 \mathrm{Ka}$ ipnim nikatpilitash ipnimain watu Ina inahtuihnu ipna Inim watu hatauitash wash.

$39 \mathrm{Ka}$ ipi ipnashapokaswisha, ipnim wakaswina pahauktatasha; Kaua taklai ipna popsiausi, Inwastki, ipnim wakaswina payaktatasha,

40 Kapam ipnim imuna ath hihimiusa, ipnimtit Ina hihimiusam. Ka ipnim Ina hihimiusa, ipnimtit pahimiusa Ipna ka Kunim Ina hishapawihnima.

$41 \mathrm{Ka}$ ipnim pahimiusa imatsinpuna imatsinpunm wanikitph, ipnimka autsatatasha imatsinpunm wasatit. Ka ipnim pahimiusa 
tsanishna titokana, tsanishnim titokanm wanikitph, ipnimka autsatatasha tsanishnim tito. kanm wasatit.

$42 \mathrm{Ka}$ ipnim yauiski kushki hishapakutatasha Kinamana naksna Kuskusana Disciplesnim wanikitph, ikuin ath hisa, ipnim watu mish shapakolwakash hiwash wasatitph.

\section{WANAHNA XI.}

K A kaua Jesusnim putimwana wah lapu1 wana Disciplesna hinashtamaluinakiya, Kaua hipakuya tuyakaiiktash, tamtainaiiktash, titauyanikashph.

2 Ka kaua Johnm pasukuananya shaktishpkinih initpkinih Christna wiakut kaua lapuana ipnim inakanikna pashapakiuya.

3 Kaua ipna pashapnishana, Imu wash Painoat, hu ma ku naksna iyohotash washi?

4 Kaua Jesus hinashmishsapailaka, hinashna sikalith atamapaikotath Johna Ki kapam wiahtaishinmka Ki kapam wiamsiaishinmka.

$5 \mathrm{Ki}$ hailatama shapasaiohohin wako hiushi; Ki haaluin shapakiaiikin wako hiushi; Ki Leprosin sapakaikin wako hiushi; Ki mimashtaps shapamsin wako hiushi; Ki titinuhnin shapautalahtin wako hiushi; Wah ki hiuituishna pauitamtainaiikshi taaishkitki.

6 Yiyaunin hiutsatatasha ka ipi watu ipnashapatakatolahtkatatasha In wasatki.

7 Ka kaua imma hipaskilina kaua Jesusnim 
hinashapnia piamhnishna Johnki ituna atk ahnaiiktaseku mimahshamih? Tukona wilatawulihlikin hatiaki?

8 Matu ituna atk ahnaiiktaseku? Naksna hamana ipnashapashamkin hamoihamoiki shamahki? Matu mimiohatom hainitpasim hiutsatanih ka ima hamoihamoiki imamashapashamkatanih.

9 Matu ituna atk ahnaiiktaseku? imatsinpuna? ikuin ath hisa imatsinpunpama hinoktisha.

10 Yoh hiwash Ka ipna pauyatimananihna; Mitsim! In tamtainatoki A shapanoktiaisha, wak ipnim haniaitataisha Kiaiikash imnokt.

11 Ikuin ath hisa ka Kalonim aiatopkinih witsat hiwash Kos watu mina naksnim Kunama panoktianya Johna Baptainawatona; sauin Ka ipi kutskutsanih Aishniwashnim inakanikitpama, Kunim panoktiaisha ipna.

12 Johnpkinih Baptainawatopkinih oko kinaph Aishniwashna taaiskna tialpash likutsama Kapshiki, Koso kapskapsnawitki hiuyatialpshi Kunia.

13 Uyikalom haimatsinpunm wah tamaluit hiuyamatsinpanihnima Johnph.

14 Kupam pamsakuanaktamh, yoh hiwash Elias, Ka ipi hipaitatashaka.

$15 \mathrm{Ka}$ ipnim matsaiyu autsatah, ipi himsitah.

16 Matu kaua ituki In ashapasatah Kinia 
naks piimna? Kushka mamaias hitamianwashpa hipatamayakaktah, Kaua lautuana hipatanuetah.

17 Hipahinah wako tuuwashki, Ath nun winpaisheka, sauin watu imanka wasasheka, kaua taklai ath nun winaisheka, sauin watu imunka wisheka.

18 Inaki John hikiaiikshaka hipaiai, ipnakuyai, sauin panaisheka Devilhin hiwash.

19 Kaua taklai hamanm Miahs hipaiksha, ipnakuiksha kaua imam panaishi ahshimilin hiwash, sisyukisna kushna kutipas, mimitsitpashna hatauitipas wah Pipublicona. Sauin wapsukuawinm ipnashapamsaiskash ipnimniki mamaiaski.

20 Kaua hinashtanania uyikalana titauyanikashna, Ka kuna hinashtituatityayanauyoyaiikshaka, immamatamaskalikshimaiki.

21 Wakainin im Chorazin awash! Wakainin im Bethsaida awash. $\mathrm{Ku}$ hinashtitwatityayanauyaiikola Tyrena wah Sidona, kapamah kush imuna kushaka; wakepa immamatamaskalikoka Sackclothki wah ilapqiki.

22 Sauin ath hisa shapatkukinwashpa, ima ath awautatashinki wakaiki Tyrena wah Sidona.

23 Kaua im Capernaum, inaki shapanoktin awash akamkinikaih Kaau, sauin animkinikaih tulahnanash awash. Tsalaui Sodomna hinashtitwatityayanauyoyaiikanioka kamah- 
kush imana Kushaka, oka taks lahaipa wakas hiutsaioka.

24 Sauin A hisa shapatkukinwashpa anashwautatasha Sodomna wakaiki.

25 Kaua Jesus ipnana kaitsiauyau, Natotam, wako kinia wiakuna anashapalukapikima wiwepsuhna wak ipnashpotahskishna Kaua taklai mimaiapkana anashapahna.

$26 \mathrm{Kushu}$ awiakuya Tota, tahs awiakuya imimnihki timiuki.

27 Uyikala patu hiniatuihnima Natotam Ina; Kaua watu ishinm naksnim titokanm pasukuasa Miahsna; ipsiwatnim Pishitpimsim: Kaua taklai watu ishinm naksnim titokanm pasukuasa Pisht, ipsiwatnim Miahspimsim; Kaua ipnimka, ka ipna Miahspim pashapasukuasa ipnimki.

28 Kaua hinashna kapam ima sapalihniksi Kapam ima tamasininkaiikshi, Kumth uyikalo inimph Kapama shapalakain.

29 Inim shapuhaikitki imamashapuhaikth, Ina tuiyakt inpukinianimth, In wah amkakais, In wa talhtalh, Kaua ath payahnu tim. naain shapalakainash.

30 Inim shapuhaikit hisauis wash, inim inakihnikt wash hisauis.

\section{WANAHNA XII.}

K AUA Jesus Discipleshinah hipakiaiika 1 pakaspa halapawitpa Ki mat, Disciples 
hayahna; Kunki pawikupshana pukumina pauyapshana:

$2 \mathrm{Ki}$ mat $\mathrm{Ka}$ kaua Pharisipum paksina, Kaua ipna pasina awah! imim inaknikt halapawitpa tamaluitkinih kipkinwhai nahship hikushi.

$3 \mathrm{Ki}$ mat hinashmishtakanpa, hinashna imat watu kos hitamasi Davidna kut, inaknikthinah, Ka kaua hipayahna?

4 Kaua paasanya Godna init, shapahnishna pakasna papshina; Matu watu awaka hipash lilkuh, wak inaknikt: Imosiwatsim titalamionawatosim hipash auyausanihnah?

5 Imat kos watu hanaka hitamasi tamaluitpa Ka kuna titalamionawat walim kutki huyakutanih, inah ipnoyiyaukinwashpa initpa, inah halahpawitpa, sauin watu shapatkukash hutsatanih.

6 Matu ath hisa, Ki naks hiwash, ipnoyiyaukinwashna inina pauwausa.

7 Kapam apasukuananyoka naks tsekin; In ashaiktatu yiyauna, wah watu talamionashnasim. Kaua wutu patananioka Kapshishwiaina.

8 Atka Hamanm Miahs hiwash, halahpawinm Lord.

9 Ka kaua Kunih hikuya kaua ipnoyiyaukinwashpa initpa hinatsasoya.

$10 \mathrm{Ki}$ mat kuna naks hama hiwaka ilakasaskin, ipshus, kaua imam pashapnishana ip- 
na toptakitash $\mathrm{Ku}$ wat tamaluitki wakas hanitash halahpawitpa hiwash?

11 Kaua hinashmitstakanpa, hinashapnia ishinm imampama otah naksni ship, kaua yoh hitkotah halahpawitpa kaua watu kunia apanakahtkitah?

12 Mish watu titokanm shipna panoktiaisa? Kunki tamaluitki shapatahswitash inaki halahpawitpa.

13 Kaua yokopna hamana pana, ipshus tukapalikim. Kaua hitkopalika, Ki mat wakas autsaya ipshus, ka Kush naka awaka.

14 Kunki Phariseepum paasapikshana patamaluioshina ipna wapsiaunash.

$15 \mathrm{Ka}$ kaua Jesus hinashsukuananya, kaua hinashniwihnana; Ki mat pauwatasnim patuiksina kaua uyikalana wakas hinashwihania.

16 Kaua hinashna, Wat mat ina apatamapaikukum.

17 Kunki hipatsanima Esaiasnim imaitsinpunm tsekaph.

18 Hihina hahnanimth ina waiimtas, ki inim shaihnin, inim hatauin wash: Kinki inim wakaswit, aishtatu, Kinia In aunitatasha inim wakaswit, .kinm Gentilepuna hinashapamsitatasha Tamaluit.

19 Watu hinatsnikotatasha, watu hiwelwasitatasha, watu ishinm kinia pamsitatasha kiaiikitpa. 
20 Watu suuisuuina tokona pauikupta. tasha, watu hilatasktatatsha ilamuhmuhnin kaimo, hinashtuwahkaiktatasha taaishkitki.

21 Ipnim wanikitpa patkaluktatashi Gentilepum.

22 Kaua ipna panahpaikoshana naks Devilhinah ilatama, tsekshimai Kaua wakas pania kalaunika ilatama hisaiohoya, tsekshimai hitsehna.

23 Kunki uyikalanm titokanm pasiwaina: pishapnia wat ki Davidnim Miahs?

$24 \mathrm{Ka}$ kaua Phariseepum pamsishana, kaua hipatsehna, kala Beelzebubkisim Didevilm Miohatokisim hinashapatatu Didevilna.

$25 \mathrm{Ki}$ mat Jesusnim hinasukuananya immuna timipniot, kunki hinashna, ka mina naks watash pituelkauin hiwash, yoh wako ipnashapaukainash hiwash ka mina naks tauyanikt, hu ma init pituelkauin, yoh wako ipnashapaukainash hiwash.

26 Tsalui Satanki Satana in shapatatu, yoh wako pituelkauin hiwash, kaua manmaii ipnim inakanikt witsatash?

27 Tsalui in Beelzebubki nashapatatu Didevilna, kaua ishinki imarn mamaiasnim pashapatanih Didevilna? Kunki immam imuna ath ishapatkuktatashi.

28 Tsalui Godnim Spiritki In anashapatatu Didevilna; atapam ikuin, Godnim aishniwashnim imuna hipainosha. 
29 Kaua manmaiih naksnim paasotah kapskapsna, initph watqaiktash wishanikt, matu ipi kapskaps walatpaiai? Kapskapsnankos polatpatah, kauana watqaiktash init.

$30 \mathrm{Ka}$ ipnim ina watu, hitqaluktatum ipnim ina tuelkauitatum; ka ipnim watu nun sapaamtanih, ipnimtit ina hitapatsaskaitatum.

31 Kunki ath hisa, titokana uyikala wiakapshishwit, uyikala wiatoptakit; Laam waunash hiwash, naksni watu waunash, ka imam Holy Ghostiph hitoptakitanih, imuna watu waunash.

$32 \mathrm{Ka}$ ipi hitoptakitatu hamanm Miahsph, ipi waunash hiwash matu ka ipi taklai hitoptakitatu Holy Ghostiph, ipi watu waunash hiwash, kina watashpa wah hanaka piamkinwashpa, watu waunash hiwash.

33 Titahs taulikit, kunim titahs timanit autsatatu; kakapshish taulikit, kunim kakapshish timanit autsatatu, kunki timanitkisim shukash hutsatatu taulikit.

34 Tukumutalikt kakan ath washi! kakapshish ath washi, kunki minmaiih tahswitph wiatsehnash ath washi, kala kunku, timnapkinih him huyatsehtatu.

35 Tahs titokan tahswitphkisim huyatsehtatu ipnim tahs timnapkinih; kaua kakapshish titokan kapshishwitpkisim huyatsehtatu, ipnim kapshish timnapkinih. $36 \mathrm{Kunki}$ ath hisa, uyikalanm titokanm 
imamatamapaiktatashi, shapatkukinwashpa, kaiashpama wiatsekin laam.

37 Kunki imam wiatsekipkisim imamashapotasktatashi; kaua taklai imamwiatsekipkisim shapatkukash ath witsatatashi.

38 Kunki tatoshmam timanawatopama, wah tatoshmam Phariseepupama pashapnishana, pana, Num Takash, kumah pahnaitah imana titwatityayanawit.

$39 \mathrm{Ki}$ mat hinashsuyatkalaika, hinashna naks piimnim kakapshishnim pipahwetipasnim, pahnipashwishi naksna titwatityayanawina, matu watu mina naksna titwatityayanawina pahtatshi; Naksnina Jonanna imatsinpuna titwatityayanawit pahnaitataishi.

40 Atka Jona hiwaka, mitat lahain Whalenim ilutpa kalaskutita; Wah kushtit, hamanm Miahs hiutsatatasha, mitat lahain watashnim timnapa wah kalaskutita.

41 Ninevehnm titokan hutalahtuktatashi, kiniin naks piimnin shapatkukinwashpa, kaua kinia pashapatkuktatashi ; atka Jonanm tamtaiki imarnatamaskalika, unatu ki naks hiwash Jonana patosksa.

42 Lokaspkinikaipu miohat, aiat, hutalahtoktatasha, kiniin, naks piimnin, shapatkukinwash pa, kaua kinia hinashapatkuktatasha: Atka lamtaipkinih watashpkinih hiuskainima Solomona wapsukuawit mitsiohotash : matu ki naks hiwash, Solomona patosksa. 
$43 \mathrm{Ka}$ kaua kapshishwinm patapiktatu ha mana, kaua hikiaiiktatu, kayauishpa, ipnashapatauyaniktash; Ki mat watu paiyaktatu.

44 Kaua ipnatatu, wakat siklitoks inim initph, kah kunih ata; Ki mat ka kaua hipaina, imat kos kaaukaau, sapsilaikin, hana. lamkin.

45 Kunki winapwana, imunatit, hinashmu. tatu, matu ipna uikinih pauwausi kapshishwitki, kauana paasotanih, kaua kuna hitau. yaniktanih: Kunki kinm hamanm lamtainim piimnim pauwautata waiat ipna uyina piimna wakainashph, kushtit kinm kakapshishnim piimnim hikutatashi.

46 Kos hinashtamtaisana titekana, Ki mat kaua ipnim pika, wak askama papainosha amti, ipna tanuetash.

47 Kaua naksnim patamapaikoya ipna, atam imisham wak imskapnim iyohoshi, A tanuetash.

$48 \mathrm{Ki}$ mat pasuyatkalaika, ipna pana, ishinah watah inim pika wak inim askama?

49 Kaua hinashtukapkaikoya inaknikt, kaua hisana, waya hahnanimht ina pika wak askama!

50 Atka ipi, Natot Akamkinikaipa tamaluitkisim, huyakutatu yohsim inim wash askap, wah kanash, wah pika. 


\section{WANAHNA XIII.}

TK UNAINI lahaipa, initpkinih hinatsataI pika, iwatam laikinpa huhshalika.

2 Kaua pauwatasnim paamhnoya ipna, kunki liashph uikinih hiwasaiya, kaua huhshalika, kaua iwahtam laikinpa pauwatashnim pohalikoya.

3 Kaua ilahinaham hinashtuyaka sapasatki : Hinashna, mitsimth, naks tamanikawat, hiwatsastsasna.

$4 \mathrm{Ka}$ kaua hiwatsastsna, tatosh lalh hitkeka katatkatatpa, kunki wayutalihnim laam papa.

5 Kaua tatosh hitkeka pishwepa, ka kuna milas ith hiwaka, kunki lamlamat hipiimna, atka saiau imit ith awaka.

6 Matu ka kaua hishamtuks hitinahta, kaua hilatahaha; atka saiau imit hash awaka, kunki hilatahaha.

7 Kaua tatosh hitkeka shishnimpa kaua ipinka shishnim hipiimna; kunki pakasna polaukaka.

8 Matu tatosh hitkeka titahspa watashpa, kunki lalahna pashapapiimna, tatosh putaptit, kaua tatosh wilaksaptit, kaua tatosh mitaptit.

$9 \mathrm{Ka}$ ipnim matsaiyu autsatatu, ipnim mitsitash ush.

10 Kaua ipnim Disciplespim pashapnia 
ipna, imat shapasatkisim anashtuyaktatu immuna?

11 Kaua hinashna atka imasim sukuanaitash Akamkinikona taaiskna paleis tsekin; matu immam watu sukuanash aushi,

$12 \mathrm{Ka}$ ipnim autsatatu, ipna initash ush kunki pauikinih ilahni autsatatasha; matu kaua taklai Ka ipnim saiau, patu autsatatu, kunia inpaitash ush ka kala patu autsatatu.

13 Kunki immuna sapasatki In anashtam. taitatu; Inaki hakinash aushi, sauin watu haksi ; Inaki mitsitash aushi, sauin watu himsashi; Watu himsukuasi.

14 Ata Esaiasnim imatsinpith kinama atsasi, Ka yoh hitsehna, mitsiohotki, inaki ath amsitatashi, sauin watu ath amsukuatatashi, inaki saiohotki atk aktatashi, sauin watu ath ashuktatashi.

15 Atka kinm ittokanm timina titiskau auwushi; Matsaiyu kilhnin auwushi; Silu kapsnin imamanin; Kaina imam siluki hishuktatashi ; Kaina imam matsiuki himshuktatashi; Kaina imam timnaki hisuktatashi ; Immamatamaskalikitph, kaina in wakas anashanitatasha.

16 Matu yiyaunin silu washi; Atka ima ath shukshi. Yiyaunin matsaiyu ath washi; Atka ima atk amshukshi.

17 Ikuin ath hisa ilahniwam haimatsinpum, wah tsitsanishnim kinia pauyahnipaswianih- 
na, Kapam kinia Kaua pain ath aksi, sauin watu paksina, kinia pauyamsiohotipaswianihna kapam kinia, kaua pain, atk amsishi ; Sauin watu pamsishina.

18 Tamanikawatona shapasat amsianith.

$19 \mathrm{Ka}$ ipnim pamsiaitatu Akamkinikona taaiskit, matu watu pasukuanaitatu, kaua ipna papainotatu kapshishnim, kaua panpaitatu ka kalapki timnaph hitkektatu; Ki hiwash, ka kuna hitkektatu katatkatatpa.

20 Kaua ki hiwash ipi, ka kaua pishwepa hitkektatu lalh, ipi himsitatu taaiskit kaua aiski hinpukinitatu ;

21 Matu saiau autsatatu ahs timnaph, kunki miwasain hiutsatatu; Ka kaua toptakinm wah talaulakinm papainotanih, kauana taaisktwasatki ipnashaptakatolakitatu.

22 Kaua hanako Ki hiwash ipi, Ka kuna shishnimpa hitkektatu lalh, ipi himsitatu, matu kaua wataspamanm keshnim wah kuiswinm talauiahtimnim polaukaktatu taaiskna, kunki saiau lalh autsatatu.

23 Kaua taklai ki hiwash ipi, ka kuna titahspa watashpa hitkektatu, ipi himsitatu taaiskna, kaua pamshuktatu, kaua hishapapiintatu lalh: Kaua uikinih shapiimtatu lalh tatosh putaptit, kaua tatosh, wilaksaptit, kaua tatosh mitaptit.

24 Kava hanaka sapasat hinatsinia; Hinashna Akamkinikom taaiskt, kuska naks 
hama hitamanika tahski lalahki ipnim tamanikashpa.

$25 \mathrm{Ka}$ kaua hipatautalika; Ki mat ipnim tuelkam patautamanikanya, pakaspa Tareski, kaua hipakuya.

$26 \mathrm{Ka}$ kaua hipiimna paks, Ka kaua hilalkiya, kauatita ipinka, Tares hiutsaya.

27 Kunki ipnim waiimtaspim papainoshina, ipna pasina imat watu tahsnasim lalahnasim atamanika kaua minih Tares hiutsaya?

28 Kaua hinashna atanm tuelkam hikianish. Kunki waimtasnim pasana watah nun apasapkiutktah?

29 Kaua hinashna watu, kainapam pakshinahna atk apopalishtaku.

30 Kuno waya ka uilapti hipiim tupinash, Kos kaua tupniwashpa, in anashnu titupnawatona, Taresna kos ath apasapaamku, ath apawaushukuiku, shapalunash; Matu paks̄ ath panikanukum inikashph.

31 Kana hanaka naks sapasat hinatsnia, hinashna, Akamkinikom taaiskt kuska Mustardnim lalh, kaua kunia naksnim hamanın panipa, kaua patarnanika.

32 Inaki kinm uyikalana lalahna hinashkatohksha kutskutsanawitki, sauin ka kaua hipiimlaulakitatu, kaua taklai uyikalana tamanikina hinashkatosktatu himakasnawitki, kalaunika patan hiutsatatu; Kalaunika ip- 
nim patishpa wayutalikin hialatiaktanih, hiwautuktanih.

33 Kaua hanaka sapasatki hinashtamtaina; Akamkinikom taaiskt Kuska shapapulamnash, Kunki naks aiat hishapatuana mit hikai tutnin, Kalaunika laam shapapulamnin autsaya.

34 Jesusnim hinashtuyaka pauwatasna, uyikalaph, sapasatki, watu mish sapasaiaiki immuna hinashtuyayaka.

35 Kunki imatsinpunm tsekaph atsana; Ka yoh hitsehna; Shapasatki in tsektatasha; in atamapaiktatasham watashnim witsatuitpkinih, paleisna wiakuna.

36 Kaua Jesus hinashwishapasiklitoka pauwatasna, kaua inith haasa. Kaua inakniktpiin pasoatuiksana, pasana nashimtakim yokapi sapasat tareski.

37 Kaua hinashmishtakanpa, hinashna, Ka Ipi hiwatsastsastatu tahski lalahki, Ipi hiwash hamanm Miahs.

38 Tamanikash hiwash uyikashli watash; Titahs lalh hushi aishniwashnim mamaias matu Tares hiushi Kapshishnim mamaias. $39 \mathrm{Ka}$ ipi tuelkam patamanikaitatu Tareski ; Ipi hiwash Devil ; Lamtaipa kaaupa hutsayu tupinash; Titupniwat hiutsatanih Haangel.

$40 \mathrm{Ka}$ kush Taresna pauyasapaamhtanih pauyashapalutanih; Kushtit lamtaipa Kaaupa hikutatasha. 
41 Hamanm Miahs hinashwishapawihnatatasha, Ipnim Haangelna; Kaua immam pauitamahtatashi ipnim inakniktpkinih, uyikalana shapakapshishwishna, wah uyikalana patokashwishna:

42 Kaua immuna patulutatashi haauph alaph; Kaua kuna hilalwahwahtatashi; Hitit kanptatashi.

43 Matu kauatita titahsnim alaukauitatashi Kuska, hishamtuks immam Pishitpim aishniwashph. Ka ipnim matsayu autsatatu ipi himsitah.

44 Akamkinikom taaiskit hiwash kuska, shaihnin imit watashpa hiwatah kaua naksnim hamanm kunia payahnah, kaua ishtakitah, kaua liloiki hisiklinah, laam patu hitamiatah, kaua kunapki watashph, hitamiatah.

45 Kaua hanaka Akamkinikom taaiskit hiwash Kushka itamiawat, Kunim papawiaiik: tah titahsnihna, sishaihnishna,

46 Kaua naksna shaikinashna payahnah, ilahniain tamiatash, Kaua hisiklinah, laam patu hitamiatah, Kaua kunapki, hitamiatah. 47 Kaua hanaka Akamkinikom taaiskit hiwash, kushka takash, kaua kunki iwahtampa hipataketah, Ki mat uyikalana patuna patakelpshanah.

48 Kaua wako kakamam, kaua kunia panakatkatah watasph, kaua hipatamaiyakektah, 
Titahsna pasapkilaktah hikaiph, matu Kakapshishna paukainah.

49 Kushtit hikutatasha lamtaipa kaaupa. Haangelm hiwiwihnatatasha Kakapshishna pankauyutatasha tsitsanishpkinih;

50 Kaua kunmana patulutatashi haauph alaph : Kaua kuna hilalwahwaktatashi, hitit kanptatashi.

51 Kaua Jesus hinashapnia inakniktna, wat ath amsukuaseka uyikalana, kapamah kala In wiahimtakshaka? Kaua pasina, A Lord.

52 Kaua hinashna, ka Ipi hiwash tamtainat wako himtakin, Akamkinikom taaiskitki, hiwash, kuska naks hama init inaknikawat hiwatah, kaua kunim panakahtah ipnim inikashpkinih, wakemana wiakuna, wah kikim. tina wiakuna.

53 Ka kaua Jesus sapasatki hinashtuyaknakia; Kaua kunih hipakuya.

54 Ka kaua ipnimnihpa watashpa hipaitoka, immam ipnoyiyaukinwashpa initpa, hinashtuyaka, ki mat pamsiswaikshana; Kaua pisina minik otah kinm wapsukuiwit wah titwatityayanawit?

55 Mish, watu ush patu haniawatom Miahs? Mish, watu ush pika Mary? Kaua mish, watu ush askama James, wah Joses, wah Simon, wah Judas?

56 Mish watu ush kanishma nunpama? Kaua minih otah ki kush wiakut? 
57 Kunki pasimkshina ipna. Kunki Jesus hinashna haimatsinpun Kanin hiutsatanih uyikalapa wiwatashpa; Naksnipa watu, ipnim watashpa, wah ipnim initpa.

58 Kunki watu mish ilahninaham hinashtitwatityayanawioya, immam talauikiph.

\section{WANAHNA XIV.}

\section{K AUATITA Herodnim Tetrarchnim pam. I sianya Jesusna wiakut;}

2 Kaua inaknikt hihina, ata hiutalahtoka John Baptainawat tinuhnishpkinih, wah yoh hiwash, Kunki ipnimki titwatityayanawit ilahni autsaiiksha.

3 Wako ata Herodnim panpa Johna, pulatpaya, shaktisph inith panika; Herodiaswasatki askapim Philipnim iwapna, wasatki.

4 Atka wako John hisana ipna watu tamaluitpama awash iwapna.

5 Kunki popsiaunipaswia ipna, matu titokana hinashsikauna; Atka immam panakshana imatsinpun ipi hiwash.

$6 \mathrm{Ka}$ kaua Herod ipnim witsat hitimipnia; Kaua Herodiasnim timaiimiaspim, hinashwasoya, pashapaaishna Herodna.

7 Kunki tamaluitki, hihina ipna, kum itu inahmunamh kaua A yoh initah.

$8 \mathrm{Ki}$ mat pikapim shapatseki hihina A initatasham Johnm Baptainawatom hushus, walatakaipa. 
$9 \mathrm{Ki}$ mat pakomna miohatom: Sauin alalpna tamaluit, wah ka ima hipshina, Kunki hinashna aunith.

10 Kaua pashapashakolka Johna shaktishpa initpa.

11 Kaua ipnim hushus walatakaipa, panahpaikoya timaina, pania: Kaua uikini pika hinia.

12 Kaua ipnim inaknikt apaina silakt patamikaishana; Kaua Jesusna patamapaikoshana.

$13 \mathrm{Ka}$ kaua Jesus himsia, kaua liashki titokannotpa wataspa hikuya: Ka kaua titokanm pasukuanatuiksana, kaua patuiksana, wihnanih titauyanikashpkinih:

14 Kaua Jesus ‘hinashwaukunia, Ki mat hinashnimakunia pauwatashna; Kaua hinashtimnayauna immuna, kikomainin wakas hinashanianya.

15 Wako hipakulawitna, kaua ipnim Disciplespim papainoshana ipna; Pasana titokanotpa kia washi, mat wako kia palahainakaun; waya anashwishapaskilitoh kinia titokana, ka titauyanikash imamaktamiatah.

$16 \mathrm{Ki}$ mat Jesus hinashsuyatkalaika, hinashna itup hipakiu ? Ima ashapapith.

17 Kaua pasana pahatih kia washi ipah wah lapit suyam.

18 Kaua hinashna inimtku.

19 Kaua pauwatashna hinashna tamayaka- 
kith pakaspa, kaua yokopna pahatina ipahna wah lapitana suyamna, paanpa, kaua hilastalasaiya Akamkinikoph, Kaua ipnoyiyauka, kaua pawitimka, kaua Disciples hinatsnia ipah, kaua Disciplespim uikinih patakasana pauwatasna.

20 Kaua uyikala papshana hipatkalaka: Ki mat putmatitpas, wah lapatitpas, pashapakakamamshana kaukaiki.

21 Pakaham paputimushus hahamsim, papshana; Kaua haatwai wah mamaias hitama. nai.

22 Kaua Jesus hinashapusaiya liasph Disciples; nakaika hinashapayahtaya immunankos, kaua ipi hinashwishapaskilitoka pauwatasna. $23 \mathrm{Ka}$ kaua hinashwishapaskilitoknakia, kaua ipsiwat hipalatia mahshamka, ipnoyiyauktash; kaua ipsiwat kunainih hikulawitnakauna.

24 Matu ipinka liash ipsiwat hiwaka lahpa iwatampa, matu hauinim pakia, atka anoktkinih hinashatianoya.

25 Kaua kaaupa Jesusnim hinashtuihna, kushpa hiwihnana.

$26 \mathrm{Ka}$ kaua Disciplespim panmakunishana ipna, hiwahyam iwahtampa, kaua hipaskauna, pisina kia hiwaplapsha, kaua sikauki hipatyuhna.

$27 \mathrm{Ki}$ mat Jesus hinashmishtakanpa, hinashna, ituain sikausi, in wash, aishith. 
28 Kaua Peter pamstakanpa, pana In Takash, tsalui im awash, kaua ina ashapawihnanukum kushpa.

29 Kaua pana Kumku, kaua Peter hitamika liashpkinih, kaua kushpa pawaukunia, Jesusna. $30 \mathrm{Ka}$ kaua pahna hatiana kapish, kaua paskauna, kunki milililh hikuya kaua hilwahwahna, pana Lord inpim.

31 Kaua Jesusnim lamlamat patkopalikoyi, ipna paanpa, kaua pana, atam kutskuts mitssukuanakt waka, ituph ashiausaka.

32 Ka kaua liashpa hipawasaya, kaua Kunmaini hiwaitalka.

$33 \mathrm{Ka}$ inma liashpa hiushina, immam pauwaukunishana ipna kaua potahskshana ipna, pasana, atam ikuin wash Godnim Miahs.

34 Ka kaua hipatkekika nakaika, kaua hipawihnankika Gennesaretnim watashpa.

$35 \mathrm{Ka}$ kaua kunamanihnim pasukuanokinishana ipna, kaua piwishapanpatana uyikashliph wiwatash, kikomainishna, panahpaikoshana ipna.

36 Ipna pasana kumak inakih shamah talam panpaitah; ki mat ka kalonim panpaishana kalo wakas hipautsaya.

\section{WANAHNA XV.}

TK AUA Jesusna papainoshana, Jerusalem11 kinih Phariseepum, wah Titimanawatom, pasana. 
2 Manama, im inakniknim hiukaisi titakashna tamaluit, atka hiuapshi ipshuski wapaikaiaiki.

$3 \mathrm{Ki}$ mat hinashsuyatkalaika, immuna ; Hinashna, manama, imanka ath aukainaishi, Godna tamaluit, imamniki tamaluitki.

4 Atka hitamaluiya God, hihina, im Totaph, wak im ishaph, akanu, ka ipi pispt hu itu pika hiwathtimoyu, ipi wapsiaunash hiutsayu.

5 Matu imam ath taklai nahshap, tamaluit washi; ima ath hitanih sapatkukaiai hiutsatatu, ka ipi hitatu pisht, hu itu pika iniin hiwash, talamionash, kam kunki imashapayiyaunah inpkinih.

6 Kunki ipnim pisht hu ma pika kanai, hatauiai, hitkautsatatu. Kunki imam tamaluinm pauyatyakalksha Godnim tamaluina.

7 Kolwakin ath washi; ataparn Tsa Esaias, imuna, himatsinpanikana, hinanikana.

8 Kala himkisim in hiuyakiutatum kinmam titokanm, himkisim ina hiuyakantatum kinmam titokanm; Matu waiat timina awiushi.

9 Kala titokanm tamaluit pauyahimtakshi, kunki immamoyiyaukliwakshi inimph titokanm tamaluitki.

10 Kaua pauwatashna hinashmuna, kaua hinashna, Mitsimth, timipnith, lauit.

$11 \mathrm{Ka}$ yoh himpa haastatu titokana, watu hiwash shapakapshishwitash; Yohsim hi- 
wash shapakapshishwitash titokana, ka yoh haatatu himkinih.

12 Kaua ipnim Disciplesnim ipna papainoya, pasana, imat watu asukusa? Phariseepum A hisiwatnaishi; Kam yoh im wiatseksaka.

$13 \mathrm{Ki}$ mat hinashmishtakanpa, hinashna, Ka yoh Natotam watu tamanikin, kunia laam wisupash hiwiusha.

14 Disciples hihina ituain amsketuasi immuna: Kala ilatama hiushi, wah pahailatama pitalkalananih. Matu tsalui pahailatamasim pitalklihliktatashi, uilapwi takatulailakash hiutsatatashi, haauph.

15 Kaua Peternm pasuyatkalaika, ipna pana, waya yokopi naks shapasat, nashhimtakim nuna.

16 Kaua Jesusnim hinashna, imat imanka mishukshimai kos washi?

17 Imat watu kos sukuasi, ka yoh himpa haastatu, ilutpasim hitkektatu, kaua halakipkisim haatatu?

18 Matu taklai, ka yoh himp kinih haatatu, yohsim timnapkinih haatatu, kaua kunmamsim shapakapshishwitash titokana.

19 Atka timnapkinihsim hiutsatatu, kapshish wiatimipnit, piwaptamaun, pahwit, pipsininpt, pahwit, mishamt, piwathtimot.

20 Kunimsim pashapakapshishwitatu titokana, matu taklai, inaki naks wapaikaiaiki 
ipshushi hipitah, sauin watu kunki ipnashapakapshishwitash.

21 Kaua kunih hikuya Jesus, Tyre laikinpa wah Sidon laikinpa pauyapainoya.

$22 \mathrm{Ki}$ mat naks aiat Canaanpu, kunama, patyuhnatwihna Ipna, pana O Lord, Davidnim Miahs ; Yiyaunim, inim timaiimas shishapitiski shapatalpin Devilki.

$23 \mathrm{Ki}$ mat watu pamstakanpa. Kunki ipnim Disciplespim pasana, waya ashapasklitoh kinia, kia hiuyatiyuhnatwiksam.

24 Kauana pamstakanpa ipna, pana kala shapawihnanin wash, Israelmsim inakniktnimsim wipelenishna.

$25 \mathrm{Kau}$ ipnim aiatom pakiuya ipna, patqaikokalikoya, pana, Lord, ilapayum!

$26 \mathrm{Ki}$ mat pasuyatkalaika, pana watu mish mimaiasna hinpaitash hipt, kaua yoh toqaitotash sikamkala,

27 Kaua ipnim aiatom pamstakanpa, pana, A, ikuin Lord, matu sikamkal shum kaukain huyapaitanih.

28 Kauana Jesusnim pamstakanpa, pana, Aiat, atam himakas imim mitsukuanakt awash: Kam kashl imim waulukt wash, kashtit yiyaun awitsatatasha. Ki mat kunainih wakas Miahs autsaya.

29 Kaua kunih hikukika Jesus; Galileenim iwatam laikin hipainakika; Kaua kushship hiqoyimna, kaua huhshalika. 
30 Kaua pauwatashnim papainoshana ipna, panahpaikoshana haaluin, hailatama, tsitsehshimai, kikupnin, ilahni, kaua kunmana laam panikaishana Jesusnim ahwepa; Kaua wakas laam hinashania;

31 Kalaunika pauwatashnim patsiwaina ka kaua pauihaksana tsitsekshimaina tsekiph, kikupnishna wakaswitph, haaluishna wihnaph hailatamana saiohotph: Kunki Israelnim. Godna potahskshana.

32 Kaua Jesus Disciples himuna; hinashna kiak anashyiyausa pauwatashna, atka mit askiu inimpa hiusheka, hipaiai ; Wah watu anashapaskilitoktatasha, immuna hipaiai; kaina hipauyalutinuhnu.

33 Kaua Disciplesnim pasana ipna, wah mananm washi hipt kalaain pauwatashain, kina titokanotpa?

34 Kaua Jesus hinashna, Matu mas washi ipah? Kaua ipna pasana, winapti ipah, kaua ku masih kikutskuts suyam.

35 Kaua hinashna pauwatasna tamayakakith watashpa.

36 Kaua yokopna winaptina ipahna wah suyamna hinipa kaua pauwitimka yokopna, kaua Disciples hinia, kaua uikinih pawatashna patakasana Disciplespim.

$37 \mathrm{Ki}$ mat uyikalo hipatkalaka: $\mathrm{Ki}$ mat winapatatpash pasapkilakshana kanikshna.

38 Ata pilapaham paputamushus, haham- 
nimsim, papshana, kaua haaiat wah mamaias hitamanai.

39 Kaua hinashwishapasklina pauwatashna, kaua ipi liashki hipainima Magdala laikinpá.

\section{WANAHNA XVI.}

K AUA Phariseepum wah Sadduceepum 1 papainoshana ipna, talauyahtimtki pasana, nashapahnim titwatityayanawit Akam. kinikopkinih.

$2 \mathrm{Ki}$ mat hinashsuyatkalaika, hinashna, $\mathrm{Ku}$ lawit ath ima hitanih haikat hikutatasha, atka hitalaliksham:

3 Kaua Mayui ath ima hitanih, taks hiwakitatasha, atka hitalaliksham. Kolwakin ath washi; kinia ipalikinasim, haikanasim, ath auyaktanih, imat watu kinia wiatitwatityayanawina auyaksi?

4 Naks piimnim kakapshishnim, pipahwetipasnim, pahnipaswishi titwatityayanawina, inaki sauin watu minia naksna titwatityayanawina hakinash aushi; Naksnina Jonana imaitsinpuna titwatityayanawit hahnaitash aushi. Kaua hinashkailonapika hinashniwihnana.

$5 \mathrm{Ka}$ kaua nakai hipatkekika; Ipnim Disciples atolaya, ata hipt.

6 Kaua Jesus hinashna immuna, imamaiiath, ashiaunanith Phariseepuna, wah Sadduceepuna, shapapulamnashph. 
7 Kaua immamanakshina, pisina, paish pakasnim titolath kia hihisa.

$8 \mathrm{Ka}$ kaua Jesus hinashmitsia kaua hinashna, atapam kutskuts misukuanakt washi, ituain pakasnim titolatph imamanakshi ?

9 Imat watu amsukuasi kos, Imat watu timipnishi ka kuna pakaham paputamushus hipatkalaka pahatiki ipahki, kaua mas itatpash pasapkilaka?

10 Kaua hanaka, ka kuna pilapaham, paputamushus hipatkalaka winaptiki ipahki, kaua mas itatpash pasapkilaka?

11 Manama watu mishsukuanaishinm tsekin, yoh kapamah hisaka, ashiaunanith Phariseepuna wah Sadduceepuna, shapa. pulamnashph, mish apamak atka ipahph hisaka?

12 Kauana pamsukuanaisha na, ata watu mish ipakain shapapulamnashph hisaka, Kala Phariseepuna, wah Sadduceepuna wiakuna hisaka.

$13 \mathrm{Ka}$ kaua Jesus hipawihnana Discipleshinah Cesareaph Philippaph, wihnapa hinashwiana, immuna manamah titokanm ina hiwaniktanih, hamanm Miahs?

14 Kaua pasana ipna, tatoshnim A hitanih John Baptainawat, hiwash; Matu tatoshnim A hitanih Elias hiwash; Kaua tatoshnim A hitanih Jeremias hiwash, hu ku naks haimatsinpunpama. 
15 Kaua immunanih hinashapnia matu ishi imanakshinm ina ipi hiwash?

16 Kaua Simon Peter pamstakanpa, pana Im awash yokopi Christ, Tinuhshimainim Godnim Miahs.

$17 \mathrm{Ki}$ mat Jesusnim pamstakanpa, ipna pana yiyaunin awash Simon, Jonanm Miahs, atka watu titokanm A hihimtaka, kala Natotam aishniwashpkinih A hihimtaka.

18 Kaua hanaka A hisa; Peter awash; kaua kina pishwepa In hanitatasha Church; kaua watu maua Animkinikom Inakniktpim pankaskilkaitataisham.

19 Kaua A initatasha Akam kinikom aishniwashnim watsakash: kam kalana olatpatatasha kina watashpa, kalatit walatpin hiutsatatasha aishniwashpa: kaua taklai kam kalana auyankapkuiktatasha kina watashpa, kalatita nikapkuikin hiutsatatasha aishniwashpa.

20 Kaua Disciplesna hinashna, wat mat minia titokana apanu, kat hiwash Jesus Christ.

21. Kaua kunih Jesus ipnanaiika Disciplesph, kutatasha Jerusalem, kaua titakashnim, wah titamtainawatom, wah titimanawatom hitoptakitatashi Ina, kaua hiwapsiautatashi, matu mitaskiupa witalahtoktatasha.

$22 \mathrm{Ki}$ mat Peter paanpa ipna patatka pana watu maua Lord; Watu kush A hikutatasha,

23 Kaua Peterna pakailonoya, pana halakiph 
witsapikimth, Satan; Shishahpitis awahs inimph: Watu atam Godna wiakut timiunaisha, kala titokanasim wiakut atam timipniaisha.

24 Kaua Jesus Disciplesna hinashna, kah ipnim ina hituihnipashwisham, ipnim kos ipnawuhuimkash ush, kaua nikatpulitash kos tamkepash ush, kaua kunimsim hiwash tuihnash Ina.

$25 \mathrm{Ka}$ ipi hiyauitatasha wakeswit, iptit hawaktatashanki wakeswit: Matu kaua taklai ka ipi hawaktatasha wakeswit In wasatki, iptit hiyaktatashanki wakeswit.

$26 \mathrm{Kuh}$ naks titokan uyikala wiwatash hinpoka, wakeswit wakaiki, mish kunki ipnashapakuishwitah? Ituph atka hiwash, atkalainash titokanm wakes witph?

"27 Hipaitatash hamanın Miahs, Pishitpim siskeiswitki, ipnim Haangelhinah, kauana uyikalana hiwasatiu $\mathrm{Pa}$ immamnihph wiakutph.

28 Ikuin ath hisa ka kala hohohasi kinama tatoshnim, wakesnim panmakunitatashi hamanm Miahsna hiwahyam Inaknikthinah.

\section{WANAHNA XVII.}

T AUA kunih wilaksipa lahaipa Jesusnim 1 hinatsnahnana Peterna, wah Jamesna, askapin, Johnin, hinatsnahqoimka mahshamka, immosiwatna, 
2 Kaua kunmanokt ipnashapautsalamka, mashtai hishishkeinis autsaya, ka kush hishamtuks, shamh autsaya haihaih tamaun ka kush hilakauit.

$3 \mathrm{Ki}$ mat ipna Mosesnim wak Eliasnim papainoshana, kaua pitanaeya.

4 Kaua Peternm pana Jesusna, Lord, Tahspa kia washi: Kupamah mitat init wihaniaitah; Naks Imana, kaua naks Mosesna, kaua naks Eliasna.

5 Kos hisana, Ki mat hishishkeinis ipalikit hinashapaiimima immuna: Ki mat ipalikitkinih hitsehnima, hinashna, ki wash inim hataunih Miahs, kinki Inashapaaishtatu: Kinia Amsith.

6 Ka kaua Disciplesnim pamsishana, kaua sikauki hipatqailokoka.

7 Kaua Jesus hinashkiyoya, hinashsapalkupa, hinashna, Hohalakaikith, wat mat sikaunanih.

$8 \mathrm{Ka}$ kaua hipahohalakaika, ki mat watu ishina titokana paksina, ipsiwat Jesusna.

9 Tamikitpa Jesusnim hinashwiana immunakapam kunia okseka, wat mat titokaph apatamapaika, kos hamanm Miahs tinuhnish, pkinih hiutalahtoku.

10 Kaua ipnim Disciplespim pamstakanpa, pasina ipna, manama titimanawat hitanih Eliasnakos paitokash hiwash?

11. Kaua Jesus hinashmishtakanpa, hinashna 
immuna A, ikuin Eliasnakos hipaitoktatasha, kaua uyikala pasapatkuktatasha.

12 Matu ath hisa, wako hipaina Elias, sauin watu pashukshina, matu inmamnihki timiuki pakushana ipna. Kushtita hamanm Miahsna hikutatashi immam.

13 Kauana ipnim Disciplespim pamshukshana, ata Johniph hihisa Baptainawatoph.

$14 \mathrm{Ka}$ kaua pauwatashna papainoshina, ki mat naks hama ipna papainoya, pahitimlikoya, pana.

15 Lord, yiyaunim ina tahatomias; Shapapelenin wash, komais shapatelpin wash: alaph hitkatolataktatu, mah kushph hitkatolutatu. 16 Inaki imim Disciplesnankos anatsnahpaikoshaka, ki mat pahtokseka shapokaswitph.

17 Kaua Jesus hinashsuyatkalaika, hinashna Hiiya! mitskuanakshimai piim atapam washi, kiswinin; Mahalah imuna wiatuanash kah wash? Mahalah inotalkash kah wash imamphki? Aunahnamth ipna.

18 Kaua Jesusnim pasimhna Devilna, kunki ipnimpkinih haatkika: Kaua kunihnih wakas hiutsama yokopi tahattahat.

19 Kaua ipnim Disciplespim immosiwatnim papainoshana Jesusna, pasana ipna, Manamah watu nu apashapaata kunia?

20 Kaua Jesus hinashna, Saiau ath washeka mitsukuanakt: Ikuin ath hisa, kapam mitsukuanakt washinah kashlka Mustardnim lalh, 
kupam apahinah kinia kimulikamna, waya kunapki kuih, kaua hiwihnanah.

21 Kinpama aatash ipnoyiyaukitkisim, hipaiaikisim.

22 Kos hilakaisina Galileepa, kaua Jesus hinashna Disciplesna, himtakin hiutsatatasha haman Miahs titokanm ipshusph:

23 Kaua ipna popsiautatashi, matu mitaskiupa shapautalahtokash hiwash. Kunki pakomtamausina.

$24 \mathrm{Ka}$ kaua Capernaumpa hipapaina, kaua Tribute hinpaiikawatom papainoshina Peterna, pasana, Watu im Takash hinatsnitatu Tribute?

25 Kaua hinashna A, Matu ka kaua initph haasa, kaua Jesusnim pasuyatkalaika ipna, pashapnia. Manama A im inakisa Simon? Ishimapkinih wiwatashpama miohatom hinpaiiktanih Customna hu ituna Tributena? Immamnihpkinih mamaiaskinih hu titokapkinih?

26 Kaua Peter hihina titokapkiniku. Kaua Jesus hihina, Mat iku kaua mamaias takatolatkin hiutsatatu.

27 Sauin ka apainish, kainanm apashapakapshishwiyu, waya kuih iwatamph, wawa tuluta, ka kunim kos suyamnim pakanpu, kunia A oalpu, him A ankakshakanyu, kaua silpsilpna auyahnu; Yok A anatsniu immuna uilapwaain imim wah inim. 


\section{WANAHNA XVIII.}

\section{耳 AUATITA Disciplespim papainoshana} 1 Jesusna, pashapnia, Ishi hinatsinoktiaisha aishniwashnim inakniktph?

2 Kaua Jesusnim kustkust miahs hinatsnahmuna, kaua kunia pashauhshalika immamnokt, 3 Kaua hinashna, Ikuin ath hisa, kapam watu shapautsalamkin witsatatashi, ka kush kustkust miahs hiwash, kaua watu manama atkaunash aishniwash Akamkinikaih witsatatashi.

$4 \mathrm{Ka}$ ipi ipnashapautsalamktatasha ka kush ki kustkust miahs, ipisim hinatsnoktiaitataisha aishniwashna inaknikt.

$5 \mathrm{Ka}$ ipnim payiyautatasha naksna kina. mana mamaiaspamana kikustkustapamana inwasatph, ipnimtita Ina ahatauitatu.

6 Kaua taklai ka ipnim pashapakapshishwiyu naksna kinamana kikustkustpamana, Mitskuanakatopamana Inimph; Ku tahs uitpa tutniwashpama imsha shapuhaikin, kaua toluin hiutsatah iwatampa lahpa, ku kaua tahs hiutsatah.

7 Kapshishwinm pashapakapshishwitatasha watashna! mat iku shapakapshishwit hiuyautsatatasha; Sauin yiyawistamuan ka ipnim hamanm pashapakapshish witatasha!

$8 \mathrm{Kunki}$, tsalui imim ipshush nim, hu itunm ahwanm hishapakapshishwitatasha imana, 
kunia A okiuku, kaua aukainu: Tahs atkau. nash wakaswinwashph, hiwash, inaki aluin, hu ma kupnin, wah watu tahs tulailakash hiwash ilatahashshimaiph alaph, inaki vilapkai ipshush hu ma uipalkai ahwa.

9 Tsalui imim silunm hishapakapshishwitatasha imana, kaua kunia imankashupanyu, imaukainanyu: Tahs imim atkaunash awash wakaswinwashph inaki tokohnin, Wah watu tahs imim tulailakash awash alaph animkinikaiph inaki uilapkai silu.

10 Imamaiiath, wat mat kinamana kikustkustpamana naks na apapalanu: Ath hisa atka, kinmam Haangelpim pulaktanih Natotaph aishniwashpa.

11 Atka wakainishna wakas hishapautsatama hamanm Miahspim.

12 Manama ima nakshi? Tsalui naksnim hamanm autsatah putaptit tsanih ship, kaua kunama naks apalenah; Mish watu kuisaptina wah kuisna hinashnawihnanah, kaua palen hipawiaiiktah mimahshamiih?

13 Tsalui paiyahnah kunia, kaua ikuin ath hisa, Ki ship hinashkatosksha Kuisaptina wah Kuisna palenaina, ipnashapalloiki.

14 Kushtita watu imam Totain Aishniwashpa timiuki, shapaukainash hiwash, naks Kinama Kikustkustapama.

15 Kaua hanaka tsalui imim piama naks si. watis hikutah, Kava A akiyotah, imosiwat 
ath pitanuetah: Tsalui A imana himsitah, yoh wako Piap wakas ahanitah.

16 Matu tsalui watu A imana himsitah, Kaua naksna awaiwitah, hu ma lapuana, Kaua atk apakiyotah, Kaua lapuam hu ma mitom tamapaikitki shapatkukash hiutsatah, uyikala wiatsekin.

17 Tsalui immuna watu hinashmitsitah Kauana A atamapaiktah Churchiph: Tsalui watu Churchna hinashmitsitah, Kaua ipna A ashapautsatah pelepele, wah Publican.

18 Ikuin ath hisa, Kapam Kalona ath auyulatpatatashi Kina watashpa, Kunmanatita walatpin hiutsatatashi Aishniwashpa; Kapam Kalona auyawipkuitatashi Kina watashpa, Kunmanatita wipkuikin hiutsatatashi Aishniwashpa.

19 Kaua hanaka ath hisa tsalui naksnipa lapu ath Kinih watashpkinih apanahmunu patu Natotaph Aishniwashph, kaua yoh ath himstakanpanyukum.

$20 \mathrm{Ka}$ mina Ina wanikitki (tukalukitki) piamhtanih Kunmana In anatsipatatu.

21 Kaua Peternm pakiyoya ipna, pashapnia Lord malham, Kah Nayasam hikapshishwiotamah ina, Kaua in kunia atolaitah? winapaham?

$22 \mathrm{Ki}$ mat Jesus hihina, A hisa watu winapahamsim; atka winaptitaham wah winapaham. 
23 Kunki Aishniwashnim inaknikt hiwash Kushka naks miohat hiwatah, kaua ipi ipnashaposatitah ipnim inakaniktphkinih.

24 Kos ipnashaposatitah Ki mat panahpaikoshana naks, otah putimham paputimushuski (sisilpsisilpki) Talentski, wasatiai ipna:

$25 \mathrm{Ki}$ mat watu ituki wasatitash otah, kaua ipnim inaknikawatom pashapatamiaitatashana ipnanih, iwapnahinah, mamiahshina, patuhina, ipnashaposatitash.

$26 \mathrm{Ki}$ mat inakanikitpim, patqaikokalikoya, ipnimki ipnoyiyauka, pana In Miohat yiyaunim ina, kosu tsa awasatiu.

27 Kunki ipnim miohatom payiyauna, pakomna, pankapkuika, wasatiai pawaunanya ipna.

28 Kaua ipi hiskalinah, kaua lautua hiwaukunitah, matu otah putaptitki (sisilpsisilpki) Penceki wasatiai ipinih: Ki mat popaukaktah ipna, panah Hamtis wasatim tsa.

29 Kaủa ipnim lautuapim patqaikokalikotah ahwapa, ipnoyiyauktah ipnimki, panah, Yiyaunim kosu tsa A wasatiu.

30 Sauin watu, sauin kaua panahnanah ipna, shaktisph inith, patulailaktah, ipnashaposatitash.

31 Ka kaua ipnim lautuapim pasukuasanah, Kaua payiyausanah, ipna kut patamapaikaitasanah immam Miohatoph.

32 Kunki ipnim miohatom pamunatuihnah 
ipna, panah shishahpitis awash, matu A waunanya imana wasatiai, imoyiyaukitph:

33 Manama Kaua watu iın uikinih im lautuana ayausaka, kamah kush imana yiyausaka?

34 Kunki ipnim Miohatom pasimhnah, kaua shishapitishph paniktah, ipnashaposatitash tsa. 35 Kushtit imuna ath hikutatasha Natotam Aishniwashpa, Kapam watu timnapkinih ima awaunaitataishi imam lautuana wiakapshishwiot.

\section{WANAHNA XIX.}

T A kaua Jesus hinashtamtainakia, kaua 11 Galileepkinih hikuya Judeanm lilaikinph, Jordan kumkunih.

2 Ki mat ilahninm pauwatashnim patuiksana ipna, kaua kuna wakas hinashnima immuna.

3 Kaua immamka Phariseepum papainoshana ipna, talauiyahtimtki pashapnishana wat hiwash hahamnim wiuinash iwapna uyikalaki siwatki?

$4 \mathrm{Ki}$ mat hinashsuyatkalaika hinashna, Imat kos watu ath hitamatanih? Ka ipnim uitpa hinashanima kala hama wah aiat hinashanima.

5 Hinashna kunki hahamnim niwihnanash hiutsatanih pisht wah pika, iwapnapkisim ipnashapalishtakash; kaua imma lapu naksni silakt hiutsatanih. 
6 Kunih watu lapu hiutsatanih atka naksni silakt hiutsatatu. Ka kala Godnim shapalishtakin hiutsatatu, watu hiwash titokanm shapawiuinash.

7 Kaua pashapnishana Ipna, matu Mosesnim tamaluitki iwapna hiuyuinanihna shapawiuinashki timashki?

8 Kaua hinashna atka imuna tiauishph tim. naph Moses hinashapawiuinakana iwapna: Wah watu mish kush uitpa hikianihna.

9 Ath hisa, ka ipi iwapna, pahweaina, hiuyuitatasha, kaua uikinih naks iwapna haniu, ipi wako pahweyu: Kaua ka ipnim kunia tinuniına panpu, ipi wako pahweya.

10 Kaua ipnim Disciplespim pasana ipna, tsalui kush hahamnim wiakutash hiwash iwapnaph, kaua watu tahs iwapnin witsatash hiwash.

$11 \mathrm{Ki}$ mat immuna hinashna, mata watu uyikalanm imuna tsekin tuihnash hiushi; kalonihnim, ka kala wako shapatuihnin hiwiushi.

12 Atka wako panaks hiwiusatanih Eunuch, kushanih pikapim ilutkinih: Kaua panaks Eunuch hiwiusatanih, titokanm shapautsat hiwiusatanih: kaua hanaka panaks Eunuch hiwiusatanih, immanih immamanin Aishniwashna inaknikna, hatauitwasatki. Ka ipnim tuihnash kinia tsehna, ipnimu patuihnu.

13 Kaua panahpaikoshana ipna kikustkust 
mamaias, shapatkopalikasatash, shapayiyaunash: Ki mat Disciplesnim patatkshana i:nmuna.

14 Katu Jesus hinashna itua atatkshi, ku hipanahnam; mat iku kushpama Aishniwashnim inaknikt hiutastanih.

15 Kaua hinashtukupalikasaya, hinashyiyauna, kaua hinashnawihnana.

16 Kaua naksnim papainoya, ipna, pana, Tahs Miohat, manah tahs koh, kaua kunki wakas witsatah?

$17 \mathrm{Ki}$ mat pana, ituain Ina waniksham, Tahs? Matu ipsiwat hiwam tahs, God: Kum wakaswina akinah, tamaluina $\mathrm{A}$ aunakaniku.

18 Kaua pana, Minia? Kaua Jesusnim pana Wat mat opsiaunu, Wat mat timnapahweyu wat mat pahweyu, wat mat apanahmi shamu:

19 Im totaph wak im ishaph akanu; Im lautuna ahatauyu, kam kush imnih imahtauitatu.

$20 \mathrm{Ki}$ mat tahatom pana wako kinia laam auyanakanikima tsa, haswalapkinih; Ituph ilapwislamsam?

21 Kaua Jesusnimpana, kum im imashapatsanah, kum siklinah, kum witamiatah kam kala patu wash, kaua kunki A nashyiyaunaiiktah yiyiyawisna; kaua shaikinash awitsatah aishniwashpa ; kaua ina tuihnamh. 
$22 \mathrm{Ka}$ kaua tahatom pamsianya tsekin, ki mat komki hiskalina: Atka ilahni kuishwit awaka.

23 Kaua Jesus hinashna disciplesna, Ikuin ath hisa, miskinih askaunash kikuishnim titokanm Aishniwashph, Akamkinikaih.

24 Hanaka ath hisa, aluis askaunash hiwatah Camel ashtainim pilhpa, wah watu aluis askaunash hiwash Godnim Aishniwashph, kikuishnim titokanm.

$25 \mathrm{Ka}$ kaua ipnim Disciplesnim pamsiaishana, komais pamsiswaikshana, pisina, Wak ishi wakas hiutsayu?

26 Kaua Jesus hinashtakahna, hinashna, inaki watu titokanm shapakutash hiwash; matu Godnimsim kutash ush uyikalaph.

27 Kana Peternm parnstakanpa, pana Mat iku nun apanwihnana uyikalana patuna, $\mathbf{A}$ tuanananih; kaua ituna apahnu?

28 Kaua Jesus hinashna kapam ima Ina tuanihniktanihinm, ka kaua hiwutalahtoktatashi, ka kaua hamanm Miahs huhshiliktatasha wahshilikasatashpa, siskeisain; kaua imanka wuhshilikastatashi wahshilikasatashpa putimtipa wah lapitipa; kaua atk apashapatkuktatashi Israelna putim watashna wah lapu watashna.

$29 \mathrm{Ka}$ ipi hinwihnatatu itu, Init, askama, kanishma, pisht, itu pika, iwapna, mamaias, itu watash, ina hatauit wasatki, ipi haktatasha 
uikinih paputaptit, wah wakeswit awitsatatasha kunku.

30 Matu ilahni inoktiawat halah hiutsatatashi : kaua taklai ilahni halakinmapama hinoktitatashi.

\section{WANAHNA XX.}

A TKA Aishniwashnim inaknikt hiwash ka A kush nuks hama, sapalihlikawat hiwaka; ipi himaikuya, hinashwaiimtatana titokana ipnim Vineyardph (tamanikash).

2 Hinashna panaks Penny ath initatasha Pa naksiph lahaiph, kaua pamstakanpshana, kaua ipnim Vineyardph hinashapasapalihlika.

3 Kaua hanaka mitatipa Hourpa hikuya itamianwasph, ki mat hinashpainoya ilahniwana, tamayakakith.

4 Kaua hinashna, kiatkith inim Vineyardph, kos ath tsa tamatkuyu, Kaua hipakiatka.

5 Kaua hanaka hikuya wilaksipa Hourpa, wah kuisipa Hourpa, kaua kushtita hinashkuya.

6 Kaua hanaka putimtipa wah naksipa Hourpa, kunapkitita hikuya, kaua hinashpainoya ilahniwana, ishi mat, hitamayakshi: Kaua hinashna Itupatamaya kshi kulawawit?

7 Kaua pasana, mat iku watu ishinm hinashwaiimtasha. Kaua hinashna, Inim Vineyardph kiatkitku; kosu tsa ath tamatkuyu,

8 Kunki kulawit pana ipnim tamalukatona, waya anashmutah waiimtasna, kam anashta- 
matkutah, halakinmapkinih kam anashwapanahnana uitpamaph.

$9 \mathrm{Ka}$ kaua halahpama hipauyapaina, ki mat pipalaks Penny hinashtamatkuya.

10 Matu ka kaua uitpama hipauyapaina, hipanaka, tosh kia hitamatkutatasha: Ki mat immunanka pipalaks Penny hinashtamatkuya.

$11 \mathrm{Ka}$ kaua wako hipanpukinia, ki mat patananishana, ka ipi tahs inina Inaknikawat,

12 Pasana, Matu Naksni Hour (liklin) kima halakinma hipakiatka, sauin nunimph $\mathrm{A}$ anashapapasha, Matu nunsim Mayuipkinih kulawawit wialatusheka.

$13 \mathrm{Ki}$ mat naksna kunamana pana; Sikstua, watu A sapkuswisha, mat iku nanm naksniki Pennyki piwaianpsheka?

14 Yok awash, iniph, kaua kuih: Kamah kush imana inisha, kushtita aunitatasha ipnanka halakinmana,

15 Mishah watu inimnihki timiuki, kutash wash, inim washanikitki ? Mat kapshish silu imaniaisha inim tahswitki?

16 Kunki halahkinma hinoktitatashi ; taklai anakunma halah hiutsatatashi.

17 Wihnapa Jerusalemph, Jesus hinashwiana Disciplesna putimwana wah lapuana, immusiwatna.

18 Kia wihnasi Jerusalemph, mitsimth; kaua kuna hamana Miahs pahimtakaitataishi tita- 
lamionawatoph wah titimanawatoph; Kaua ipna patamaluiotatashi wapsiaunash,

19 Kaua ipna paniktatashi, Gentilepuph wapilaptainash, wah wawianash, wah nikatpulitash: Matu mitaskiupa hiutalahtoktatasha. 20 Zebedeenm mamaiaspim pikahina, ipna papainoya, pahitimlikoya, naksiph panahmuna ipna.

21. Kaua ipna pana, ituph inahmusam? Kaua hihina ki inim lapit mamaias wash, kum imim Miohatonawitph imashapatamalishtaktah palkai; naks washupkinikaih, naks sahiahpkinikaih.

$22 \mathrm{Ki}$ mat Jesus hinashsuyatkalaika, hinashna, watu ath sukuasi, kapam kunapki inahmusinm. Mish imanka imamakutash kunkitita hikaiki, kahkunki In inakutatasha? Mish imanka imamashapabaptainash Kunkitita Baptaiki, kah kunki In inashapabaptaitatasha? Ki mat pasana, A, Kushu kutash washi. 23 Kaua hinashna, atapamu imamakutatashi inim hikaiki, atapamu imamashapabaptaitatashi Kunkitita Baptaiki, Kah kunki, In inashapabaptaitatasha. Wah watu inimnih inashapatamalishtakash, inim wapshuhpkinikaih, hu ma sahiahkinikaih; Ush Na-Totamsim shapatamalishtakash Kalona, ka kalapki wako shapunikoin.

$24 \mathrm{Ka}$ kaua putimwam pamsishana, Ki mat pakatamnoshana lapu askap. 
25 Matu Jesus hinashmuna immuna, hinashna, wako ath sukuasi, Gentilepum mimiohat pawimiohatoiyotanih inaknikna, Titakashnim paskauwiswiotanih immuna.

26 Wah watu mish kush ath kutatashi: Matu ka ipi imampama, ipnashapanoktitapashwiyu, ipi, imuna wapaiatat hiutsatatasha, 27 Kapam ipnim, imampama, himiohatoiyotipashwiu, ipi, imam hashwalaia ath witsatatashi:

28 Atka hamanm Miahs watu mish ipnasha. popaiatatama, titokaph, kala ipisim hinashwapaiatatama, wakaswina paniktama, ilahniaintamitokash.

$29 \mathrm{Ka}$ kaua Jerichopkinih hipawihnana, kaua pauwatashtamaunishnim patuihsina ipna.

$30 \mathrm{Ki}$ mat lapu hailatama hipatamayakekaiskitpa ; kaua Jesusna pamsiokinia, kaua pa, tiuhnatkshana, Lord! Davidnim Miahs; nashyiyaunmi.

$31 \mathrm{Ki}$ mat pauwatashnim patatkshana immuna, pasana, shauth; Ki mat, uikinih hipatiuhna, pasana, Nashyiyaunim; Lord, Duvidnim Miahs!

32 Kaua Jesus hiushalika, kaua hinashmuna, hinashapnia, itunapamah kiaisha?

33 Kaua pasana Lord kum nashapasaiohotamh.

34 Kaua Jesus hinashyiyauna, silu hinash- 
wapasanya, Ki mat kunmainih hipasaiohoya, kaua patuihsana ipna.

\section{WANAHNA XXI.}

WAKO Jerusalemna pakakamnishina, Bethphagepa, Olive shawaiishpa mahshampa, wako hipapaina; Kaua Jesus hinashwaiimtaya lapiuana Disciplesna,

2 Hinashna, imamkunika tauyanikash, kuth, kunn ath payahnu. Ass, katatin, talkalikin; kaua ath apankapkuiku, ath apanahpaikoyukum Ina.

3 Tsalui ishinm ath hinu, itua akesksi? Kaua ath apanu, Kala Lordnim kinia pahwituisha, kaua ath hipashapanahnanukum.

$4 \mathrm{Kinm}$ wiakunm pashapatsanima, imatsinpunm, tsekaph; Hihina,

5 Waya Siona Pahapa autath, Mitsimth, wako im Miohatom hikakamnisham, talhtalh hiwash, Assph hiwasasham, Assnim miahsph.

6 Kaua Disciples hipakuya, ka kush Jesus hinashna kushu hipakuya,

7 Kaua panahpaikoshana Ass miahshinah, kaua immam shamh pausutakaiyashana, kaua ipna pashapshishana.

$8 \mathrm{Ki}$ mat pauwatashnim, immam shamh iskitpa panaushinikaisana; Kaua tatoshmam piskona pawaukukshana, kaua kunia iskitpa pahaushinikaisana.

9 Ka immam panoktiaishana wak immamka, 
ka immam patuihsina, uyikalonm aishki, potahskshina ipna, pasina, Hosanna Davidnim Miahs! Yiyaunin, kanm ipnim, Lordnim wanikitki, hikakamnisham; Hosanna toshnih!

$10 \mathrm{Ka}$ kaua Jerusalem hipaasa, Ki mat laam tauyanikatom patkaisana, pishapnishina, Ishi hiwash?

11 Kaua pauwatashnim pasana, Kat hiwash Jesus, yokopi Nazarethpu Imatsinpun, Galileepkinih.

12 Kana Jesus hinatsasoya Godnim Templeph (Inith,) kaua hinashsuyalahta uyikalona, Ka kalo pitamiashina Templepa, silpsilpna, Taklainawatona, pitkalainiwash hinashtamashau kulikanya; Koinu itamiawatona washshilikasatas hinashtamashaukulikanya;

13 Hinashna wako timanin hiutsama, Ina inith hiwanikaitataishi ipnoyiyaukinwash hiwash; mat atapam wako pahwenwash apanianima.

$14 \mathrm{Ki}$ mat hailatamam wah haaluishnim, papainoshana ipna. Templepa, kaua wakas hinashania.

$15 \mathrm{Ka}$ kau titalamionawatom, wah titimanawatom pahnaishana ipna titwatityayanawit, Ka kaua hipamsia mamaias, imat potahskshi, Templepa, pasina, Hosanna Davidnim Miahs! Ki mat pasiwatsana, pahehsamnoshana,

16 Pasana ipna, imat watu im anashmitsisha kinmana tsekiph? Kaua Jesus hinashmish- 
takanpa, hinashna, A, Imat Kos watu ima hitamasi kunia tsehna? Kat wako A im imashapotahskima ipauwanishki, miapkauitki?

17 Kaua hinashniwihna, tauyanikashpkinih hinatsatapika, Bethanypa hipaina; kuna hiwawituka.

18 Kaua Mayui hiskalitoka tauyanikashph, Ki mat hayahna.

19 Kaua ata pahna Fig-shauaina naksna, kaua hinaka, kah inakatah, Ki mat pahauka, kala piskosim awaka: Kunki kunia taulikina puyuwanika, watu A timanit wisatatasha. $\mathrm{Ki}$ mat Kunaini hilatahaha yokopi Fignim taulikt.

$20 \mathrm{Ka}$ kaua Disciplesnim paksana, Kaua hipatsi waina ; pisina, Ata lamlamat Fignim taulikt hilatahaha.

21 Kaua Jesus hinashsuyatkalaika, hinashna, Ikuin ath hisa, kapam mitsukuanakt washinah, kapam saiau talauikin washinah, kaua watu mish kiniasim kutash ath washinah, ka kush Fignim taulikina akushaka; Kapam apahina kinia mahshamna, waiat witsaih, imatoloih iwatamph, kaua kush hikotah.

22 Kapam kalaki imamoyiyauktah mitsuknanakaikisim, kalana laam ath aktatashi.

$23 \mathrm{Ka}$ kaua Templepa haasa, Ki mat titalamionawatom wah titokapama titakashnim, papainoshana, matu kos hinashimtakshana kaua pashpanisha na ipna, Ishinm tamaluitki 
ki kush kusha? Ishinm hishapakusha ki kush?

$24 \mathrm{Ki}$ mat Jesus hinashsuyatkalaika, hinashna, Inku ath naksip shapnisha, kapam imankos pahinam, Kauana taks ath halah hinu, kat ipnim tamaluitki, ki kush In kusha.

25 John Baptait, minih awaka, Akamkinikopkinih, hu ma titokapkinih? kaua immamatashiauka, pisina, kanm nu hitatashi, Akamkinikopkinih, kaua kia hisuyatkalaiktatasha, wah manama kaua watu apamsukuanaka ipna?

26 Tsalui nu hitatashi, Titokapkinih? Kaua kia askautatashi titokana, mat iku uyikalanm pasukuaseka, hiwaka imatsinpunm John.

27 Kunki Jesusna pasina, watu kia sukuasi. Kauana hinashna, Inka ath watu himtaksha, ka Kunim tamaluitki In Ki kush kusha.

28 Sauin ath shapatimipnisha, naksnim hamanm awaka, lapit mamaias, titahat; Naksna poyimtaiya, pana, Nisu, taks awapaiatayukum ina Vineyardph.

29 Kaua hihina, watu; Ki mat miwaspa ipnatamaiataia, kaua hikuya.

30 Kaua hanaka naksna poyimtaiya kushtit pana. Kaua pamstakanpa, pana A Tota, kosu kutatasha; ki mat watu hikuya.

31 Kaua ath shapnisha, kinmam lapiuwam, minikum pisht tsekin patuihnanya? kaua pasana, anakonmanu. Kauana Jesus hinashna, 
Ikuin ath hisa, ki Pipublicansnim, wah ki pipshitimtipasnim ath hiwausi Godnim Aishniwashph.

32 Atka John imuna hinashpainoshaka tukuhnawitki, sauin watu ath amsukuanaksheka ipna: Immamsim Pipublicansnim wah pipshitimtipasnim pamsukuanaksheka ipna: kaua imanka imamatamaskalikaiai, mitsukanakai ath washi, inaki ath kunia sukuasheka33 Wah hanaka naks sapasat, mitsianimth: Hiwaka naks inina Inakanikawat, kaua ipi hania Vineyard, kaua yokopi hishuliklika shishnim shauaiki hipalkalina, Kaua hania tamapaikash Wineain, walahnash hania, kaua hinashapanakniknihnana yokopi Vineyard, kaua waiat watashph hikuya:

$34 \mathrm{Ka}$ kaua wako atitatashana, kaua waiimtaski hinashapakioya Inaknikawatona, kunama hipt hinashapanpatana.

$35 \mathrm{Ki}$ mat Yokomam Inaknikawatom Payakaswishana waiimtasna, naksna pawawiasana, naksna popsiausana, naksna pishweki patotpiakshana.

$36 \mathrm{Ki}$ mat hanaka waiimtas hinashapawih. nanoya: Ki mat immunanka kushtit pakiukinishana.

37 Kaua lamtai hinashapawihnanoya miahs, hihina, kala hikanaitataishi Ina miahs.

$38 \mathrm{Ka}$ kaua Inaknikawatom panmakuniaishana Miahs, Kaua pisina ki hiwash Inakani- 
kash, Ka apopsiaun, Ka apanpanish, ki uyiyala, Kunanm apautsatah nunimnih.

39 Kauo panpukinishana, Vineyardkinih $\mathrm{Pa}$ tulahtsana, popsiausana.

40 Ka kunim Miohatom ush Vineyard, ka kaua hisukuatatasha, kaua manama hinashkutatasha kunmana Inaknikawatona?

41.Kaua pasina hinashwaukaitatashu shapiltuihki kunamana pipatokashna hahamna: Kaua taklai, ipnim Vineyard, nashapa Inakanikawatona hinashapanakniktatasha, ka minm pauyanitoktatasha kunama hipt tsaa.

42 Kaua Jesus hinashna, Inat watu ath kos hitamasi tamaluitpa? Yokopna pishwena, ka kunia haniawatom papalana, yohtit wako shaihnin hiwitsash, initain tulikasatash: Kala Lordnim sahpakut hiwash; matu nu kunia atsiwaisi.

43 Kunki ath hisa Godnim Aishniwash ath hihitoktatasha, kaua taklai naksip watashph hinashapanakniktatasha, Ka kunmam tsa panakniktatashi.

$44 \mathrm{Ka}$ kalo kinaph pishweph hitkatsiliktatasha, kalo hitkakuptatashi: Matu ka kalona kinm pishwenm hinashimsatatasha, laam kunama hinashsimilahtatasha.

$45 \mathrm{Ka}$ kaua titalamionawatom, wah Phariseepum pamsiaishana ipna shapasat, $\mathrm{Ki}$ mat pamshuksana, ata nunimph hitseksam.

46 Inaki panptipashwishana ipna, ki mat ti- 
tokaph paskaunoshana, atka hinakshina Imatsinpun hiwash.

\section{WANAHNA XXII.}

YUA hanaka Jesus hinashsuyatkalaika 1 immuna, sapasatki hinashtamtaina, hinashna.

2 Akamkinikom Aishniwash hiwash, kushka naks Miohat hiwaka, ipi miahs Tamasana hipt panianima.

3 Kaua waiimtasna hinashwishapanpaiika, yoh ka imma wako tamapaikoin hiushina Tamasam hipt: Ki mat watu hipakuma.

4 Kaua hanaka nahshapna hinash waiimtaiya, Hinashnatuihnna, tamapaikoin ath apatamapaikoyu, wako In hinakia hipt, wako wiwapsiaunin hiwiushi inim titalonot, wah titishkau, laam wako hinakin: Kauo kumth Tamasam hipitph.

$5 \mathrm{Ki}$ mat pasapinmishana, hipakailonapikshana Immamnihph wiakutph, naks ipnim tamanikash hikuya, naks ipniın itamianwash hikuya.

6 Kaua tatoshma paanpshana waiimtasna, payakaswishana, kaua popsiauna.

7 Kunki ka kaua Miohat hisukuana, kaua hinashehsamnoya immuna: Kauana ipnim piwapsiaunatona hinashapawinu noya, hinashapopsiautana immuna piwaptamaunipasna, hinashapalunaitana immuna tauyanikash. 
8 Kaua hanaka waiimtasna hinashna: Mat iku wako hinakin Tamasam hipt, matu ka immam, uitpa munin hiushina, watu painotash.

9 Kauo awipawith uyikalapa iskitpa, kapam kalona ath apayahnu, kalona ath apashapawihnanyukum Tamasam hipitph.

10 Kunki immam waiimtas hipawiwihnana uyikalapa iskitpa, ka kalona pauyaksana, kalona pasapaamsana, kakapshishinahna wah titahshinahna: Kauana wipainatom paasoshana Tamasam hipitph.

11 Kaua yokopnim Miohatom, hinashasoya wipainatona hahnash, ki mat naksna hamana hahna ata awaka saiau shamh Tamasain hipash :

12 Kaua pana, sikstua, manama kinaph asima, matu saiau awash shamh Tamasain hipash? Ki mat watu pamstakanpa.

$13 \mathrm{Kunki}$ waiimtasna hinashna, waya anpith kinia, ulatpath, Ankatahkaikith, Kunikuain shaktishph atulailakith: Hilalwahwahniwash, hitit kanpniwash.

14 Mat iku ilahni wimunin, sauin milas shaihnin.

15 Kaua Phariseepum paatapikshana, patimipnioshana ipna shapokapaptash, talauiah- timki, ipnim wiatsekiph.

16 Kunki immam Disciples hinashapawihnanoya, Herodpuhinah, pasina ipna, Miohat, 
wako A sukuanaishi imana wiakut, tukuh awash, Godnim wiakutki tukuh anashimtaktatum, sikaunai titokaph, mat ikum watu titokaph A imashapaktatu.

17 Waya nashnanim, manama im inakisha? Kuh initash washi Tribute Cesarna, hu kuh watu?

$18 \mathrm{Mutu}$ Jesusnim hinashsukuananya immuna patokashwit, kaua hinashna, kolwakin ath washi, itua Ina iyalasinm?

19 Waya shapahnimth silpsilp Tributeain. Kaua pashapahsana naks Penny.

20 Kaua hinashapnia, matu ishinm ush ki tuahasat, wah ki shapahnash?

21 Kaua pasina Cesarnm ush. Kaua hinashna initashu ath washi Cesarna, ka kala patu Cesarnm ush, kushtit initashu ath washi Godna, ka kala Godnim ush.

$22 \mathrm{Ka}$ kaua pamsishana, ki mat patsiwaisana, panwihnasana ipna; kaua hipawisklina.

23 Kauatita lahaipa, Sadduceepum papainoshana ipna, ka imma hinanihna saiau hiwash witalahtokt, kaua pashapnishana ipna,

24 Pasana, Miohat, Kush Moses ata hinkana, tsalui hama mimiahsnot hitinuhna, kaua askapim iwapna hiskiunianyu, kaua mamaias pashapautsanyu.

25 Winapwa hiushina askamatan nunimpa: Kaua takash iwapna hania, ki mut mimiahs- 
not hitinuhnapika, askap iwapna hinwihnanya:

26 Kaua takesh tamalukt hiskiunianya, kalaunika uyikalo hipauyaskiunia, Kalaunika uyikalo hipauyatinuhnapika mimiahsnot.

27 Kaua halah ipinka Aiat hitnuhna.

28 Matu witalahtokinwashpa minikunm iwapna autsatatasha, atka uyikalo hipauyaskiunia ipna?

29 Kaua Jesus hinashmishtakanpa, hinashna, Atapam watu sukuasi Tamaluit, wah Godna kapskapsnawit, kunki ath imamashapapelesi.

30 Atka witalahtokinwashpa watu iwapna pihanitash hiutsatatashi, kala ka kush Godnim Haangel, Aishniwashpama hiutsatatashi.

31 Imat watu kos ima hitamasi yokopi tsekin, witalahtokitpaain tinuhnishpkinih, yoh kapam Godnim hitanuesham? Hihisa,

32 In Wash Abrahamnim God, wah Isaacnim God wah Jacobnim God? Mat iku God witsatash wiwakesnimsim God, wah watu titinuhnishnim.

$33 \mathrm{Ka}$ kaua pauwatashnim pamsishana, kaua patsiwainaishana ipna tuiyakt.

34 Matu ka kaua Phariseepum pamsishana, ata wako Sadduceepuna hinashapashaun kaua imamka paamhnoshana ipna. 
35 Kaua naks timanawatom immampama, shapinauitash, pashapnia, pana ipua,

36 Miohat, ki tamaluit, wah mah hinoktisha kinama?

37 Kaua Jesusnim Pana, Lordna imim Godna, ahatauyu uyikashliki imim timnaki, uyikashliki imim wakaswitki, uyikashliki imim timiuki.

$38 \mathrm{Ki}$ hiwash takash tamaluit, ki hinoktisha. 39 Kaua naks hanaka kushtit, Im lautuana ahatauiyu kam kush imnih imahtauitatu.

$40 \mathrm{Kinm}$ lapitim tamaluinm potolasashi uyikalana tamaluina wah imatsinpuna.

41 Kos paamhnoshina Phariseepum, kaua Jesus hinashapnia,

42 Hinashna, manama ima atk inakshi Christna? Ishinm miahs hiwash? Ki mat pasina, Davidnim ush.

43 Kaua hinashna, matu kaua manama Davidnim Holy Spiritki puniksha in Takash hiwash, atka hihisa,

44 Lordnim pana in Takashna inim wapshuhpkinikai wahsulishtakim, kos im tuelkana ashapotiksatatasha.

45 Tsalui Davidnim patatu ipna in Takash, kaua minmaiih Davidnim miahs otah?

$46 \mathrm{Ki}$ mat watu ishinm naksnim mishtakanpash hiwaka; kaua kunih lahaipkinih watu ishinm naksnim sishuiki shapnitash ipna patuph hipauyautsama. 


\section{WANAHNA XXIII.}

T. AUA Jesus hinashtamtaina pauwatashna 11 wah ipnim Disciplesna,

2 Hinashna ki titimanawatom, wah ki Phariseepum pauhshilikaisaishi Mosesna wahshilikasatash :

3 Kapam kala imma hiuyahimtaktatashi, kuniasim ath auyatuihtatashi; wah wat matimmamnih wiakut apauyatuihnanyu: Kala hiuyatsehsimtanih, wah watu hiuyakutanih.

4 Atka sisininish ishaps paniatanih, Shapatamaushaliktash, kaua yokopi pauwitulikasaitanih titokana huhuipa; matu immam watu pasapatalkaitanih inaki naksniki ipshuski.

5 Atka imma hiuyakutanih immamashapahnashsim Titokaph: titilu Phylacteries immaniatanih, kikohat iskatkatin immamaniatanih,

6 Pawauluktanih titakashaina wahshilikastashna Hipinwashpa; wah titakashaina wahshilikasatashna ipnoyiyaukin washpa hainitpa.

7 Immashapashaihtanih haitamianwashpa; Immamashapuniktanih, Rabbi, Rabbi,

8 Wah wat mat imamashapanu Rabbi: Atka wako naks Rabbi ath washi, Christsim; kaua imanka askamatan ath washi.

9 Kaua wat mat ishina watashpamana apahimiunu, Tota; atka Pishit wako ath wishi, ka ipisim Aishniwashpa. 
10 Wah Wat mat Imamashapanu Miohat, atka Miohat wako ath washi, Christsim.

11 Kapam ipnim hinoktiaitapaswiaisha imuna, ipi imam waiimtas hiutsatatasha.

$12 \mathrm{Ka}$ ipi hinashkatosktapaswiu, ipi shapaksainin hiutsatatasha, kaua takalai ka ipi ipnalapwislamnu, ipi shapakatoskin hiutsatatasha.

13 Matu ima ath wakainin washi titimanawat, wah Phariseepu, kolwakin ath washi; Mat ikupam ima okalkapiktanih aishniwash titokana: Mat ikupam watu imanih Aastanih, kaua ka imma Aastipaswishi, kunmananka ath atyakalktanih.

14 Ima ath wakainin washi titimanawat, wah Phariseepu, kolwakin ath washi! Mat ikupam haawina inith ath anpaiikaitanih, imamashapahnash kohat imamoyiyauktanih: Kunki uikinih wakainash, komais shapalunash ath washi.

15 Ima ath wakainin washi titimanawat wah Phariseepu, kolwakin ath washi! Mat ikupam uyikashlipa wiwatashpa, wah uyikashlipa iwatampa ath akiaiikotanih naksip titokap imamashapoyikotash; matu kapam kaua hiwaiyikotatu imuna, kaua uikinih ath ashapakapshishwitanih ipna, imuna ath hinoktiaitatu wakainiwashph.

16 Im ath wakainin washi, hailatama inakanikawat ath washi, mat ikupam ath hitanih; ka ipi ipnanahkiuyu Templeki, ipi watu tuih. 
nash ; matu kaua taklai, ka ipi, ipnanahkiuyu Templem Goldki ipisim tuihnash hiwash.

17 Atelpin ath washi, hailatama ath washi! Mahi tosh, ki Gold, hu ma Temple ka ipi Goldna shapahautnash hiwash?

18 Ath hitanih ka ipi ipnanahkiuyu talamionwashki, ipi watu tuihnash hiwash; matu kaua taklai, ka ipi ipnanahkiuyu talamionwashnim talamionashki, ipisim tuihnash hiwash.

19 Atelpin ath washi, hailatama ath washi! Mahi tosh, ki talamionash, hu ma talamionwash, ka ipi talamionhshna shapahautnash hiwash?

20 Kush hiwash, ka ipi ipnanahkiutatu Tulamionwashki, ipi ipnanahkiutatu talamionwashki, wah talamionashki.

21 Kaua kushtit ka ipi ipnanahkiutatu Templeki, ipi ipnanahkiutatu Templeki, wah Ipnimki, ka ipi Templepa hitauyaniktatu.

22 Ka ipi ipnanahkiutatu Akamkinikaiki, ipi ipnanahkiutatu Godnim wahshilikasataski, wah ipnimki, Ka ipi Kuna huhshilikasatatu.

23 Ima ath wakainin washi titimanawat, wah Phariseepu, kolwakin ath washi! Matu ikupam ath putimptwitanih tiwantiwanki, wah aniseki, wah Cimminki : matu titluna tsekna ath awautanih tamaluitpamana, tukunawina, wah yiyauna, wah mitsukuanakna: Kiniakos 
ath akushinah, inaki watu kunia hiwash waunash.

24 Ailatama inakanikawat ath washi! Matu ath tiptipna ahautanih, matu kaua Camel ath amushtanih.

25 Ima ath wakainin washi titimanawat, wah Phariseepu, kolwakin ath washi! Mat ikupam tushkinikaisim ath sapakaiktanih, hikaina wah kiwautakaina, matu imitkinikai wikakamamnin awiushi sapkuswit wah patokashwit.

26 Ilatama Pharisee! Imitkinikainakos hikaina wah kiwantakaina asapakaiku ; kauana ikuin tushkinikainika sapakaikash hiutsayu. 27 Ina ath wakainin washi titimanawat wah Phariseepu, kolwakin ath washi! Mat ikupam ath washi ka kush haihai shapautsin tinuhnishain inikash inaki tushkinikai titahs, matu wikakamamnin hiwiushi tinuhnishnim pips wah atawatit.

28 Kushtit ath washi tushkinikaisim imamashapatsatanih titokaph, matu imit timnapa wikakamamnin ath washi, kolwakt wah patokashwit.

29 Ima ath wakainin washi titimanawat wah Phariseepu, Kolwakin ath washi! Mat ikupam ath haniaitanih haimatsinpuna tinuhnishain wianikash, ath auyalaulimkaitanih tsitsanishna tinuhnishain wianikash, 30 Ima ath auyatanih Kuh nun pauyapiim- 
noka, Ka kuna nunim pishitma hipauyapiim. nima, kaua watu nunim pishitma pauyapayoka haimatsinpuna wapsiauph.

31 Matu imamnih ath imamasukuanaishi, Ka immam pauyopsiaunanihnima haimatsinpuna, Kunmamtita mamaias ath washi.

32 Imam totaph wiakut ath auyatsanaitataishi.

33 Tukumutalikin ath washi, Vipernm Kakan ath washi! Wah manmaiih apaulalihnapiku ilatahashshimaina alana Animkinikaih?

34 Kos ath imuna wiashapakiotatasha Haimatsinpunki, matu kaua tatoshmana ath au. yopsiautatashi, tatoshmana ath auyawawiatatashi, Kalaunika ath auyopalamktatashi tatoshmana nakskinih tauyanikashpkinih, uikinih naksiph tauyanikashph.

35 Kalaunika imunasim ath hishimsitatasha Tsitsanishnim Kikanm, Ka kalapa, tsitsanishnim Kikat hiwiwalama uyik ashlipa wiwatashpa, tsanishkinih Abelm Kikatkinih uikinih oko Barachiasnim miahspim Kikatph Zachariasnim Kikatph, Kapam ipna opsiauseka Templem Talamionwashnim hapai, Kalonim Kikanm imunasim ath hishimsitatasha.

36 Ikuin ath hisa, Kinm wiatsehnim, wiakunm uyikalanm hauitukitatasha Kinia piimna ath.

37 Hiiah Jerusalem, Jerusalem, Im A auyop- 
siaunakana haimatsinpuna, A auyatotpiahkana immuna, Kam Kaloki hiyashapakiorra, A imana mamaias wiaksaluioaitapash wiaishaka, Ka kush washwashno mamaiashph hiksaluitatu waptashki, matu im A watu!

38 Wah mitsim, wako awash imim Init nikaskilkin!

39 Ath tanuehnasa, watu maua ath haktatashinm Ina; Kalaunika Kush ath hitatashinm, Yiyaunin hiwash, Ka ipi Lordnim wanikitki hikakamnisham.

\section{WANAHNA XXIV.}

I AUA Jesus hinatsatapika, Templepkinih 1 hikuya, Ki mat Disciplespim patuihsana, pashapahna ipna Templepama hainit.

2 Kaua Jesus hinashna, watu ath ima wiaksi ki uyikala? Ikuin ath hisa, nikaskilkash Ki uyikala, watu mina lapit pishwe pitulikasin hiutsatatasha.

3 Olive hishpa mahshampa, huhshalika ipi, kaua Disciplesnim papainoshana ipna pasina, waya nashimtakim, maua Ki kush hikutatash? Itunma imana Kakamnit hinashtanapaikotatasham, Itunmah watashna lamtain Hinashtamapaiktatasham?

4 Kaua Jesus hinashmitstakanpa, hinashna, Imamaiath titokaph, kainapam hipashapapelenu ath.

5 Atka ilahniwa inim wanikitki hiuyakiaiik- 
tashi, hitatashi, In wash Christ; Kunki ilahnina pashapapeletatashi.

6 Kosu ath amsitatashi piwapsiauph, wah pitamaluioth piwapsiaunash: Wah wat mat Kunki imamakesknu: Kosu laam shapatsanash hiwash, unatu watu Kunaitit hilaın. tainu.

7 Atka Kos wiwatashkinih pitilkainotatashi, wah mimiohat inakanikithinah pitokalatatashi: Hayahnim wah titoshinm pakiaiikotatashi, ilahnipa wiwatashpa, ilahni wiwatash hiwayatuwuktatashi.

8 Sauin kinm wiakunm kata mah Kus shapahisiaukash hiutsatatasha.

9 Kauana hinatsnahnatatashi ath hashwalawith shapopilaptainash, kaua ath opsiautatashi: uyikalapkinih wiwatashkinih ath hahehsamnotatashi, inim wanikit wasatki.

10 Kaua ilahniwa immamashaptakatolatkitatashi, pitamapaiktatashi, Pihehsamnotatashi.

11 Kos ilahni Mimishami haimatsinpun hikiaiiktatashi, ilahniwana pashapapeletatashi yokomam.

12 Patokashwinm Pauyashapalatahashkaitataishi ilahniwana hatauit.

13 Matu Ka ipi ipnashapahtoktatasha lamtaipa, ipisim wakas hiutsayu.

14 Mat iku Kinm Aishniwashnim taaishkitki pawitamtainaiiktatashi uyikashliph wi- 
watashph, immamain shapasukuanash; kauana hilamtainu.

15 Kapam aktatashi hautnishpa, hisiaukawatona shishapitiski ipnopalihnin, Ka kunia Danielm imatsinpunm patamapaika, (Ka ipnim pahitamanah, ipnim pamshukitah:)

16 Kauana wilalikith Judeapkinih mimahshamka:

17 Ka ipi init tushkinikaih hiutsayu, wat mat aasoyu inith, patu inahwoinash :

$18 \mathrm{Ka}$ ipi tamanikashpa hiutsayu, wat mat kalaunoyu shamh inptash.

19 Hatukas ka imma haiyah hiutsayu, wah immanka ka imma shishapakohin hiutsayu.

20 Kush imamoyiyauktatashi, "wat mat anim hu ma halahpawitpa A Shapawuitata. sham."

21. Kaua pain sisikauishnihnim shapopalaptainashnim hiutsayu, watu maua kush hiutsama watashnim anoktkinikaikinih, wah watu maua hanaka kush hiutsayu, kaua pain naksima hiutsayu.

22 Tsalui watu yokopi wialahain hinashapamilaswiotah, kaua watu mina naks titokan wakas hikokautah: matu ipnim Shaihnishwasatki hinashapamilaswiotatasha yokopi wialahain.

23 Kapam kuna naksnim titokanm ath hitatasha, ki hiwash Christ, hu ma kuna; wat mat kunia apamsketuanu. 
24 Atka ilahni mimishami Christs, wah ilahni minishami haimatsinpun hiwikiaiiktatashi, hitsakeswiaiiktatashi, hiwtitwatityayanawiaiiktatashi, Kalaunika shishaihnishna witas pashapapeletatashi, matu kala watu shapapelenash hiushi.

25 Mitsimth, hainapam washina wako ath himtaksha.

26 Kapam ath hitatashi, kat kuna hiwash ipi titokanotpa watashpa; wat mat apatuihnu: hu ma hitatashi, waya ahakin kini ipi tushti peleispa initpa; wat mat apamsketuanu.

27 Atka tinahtitkinikaikinih hitkasaiohotatu lalamat naksima Hitkektatu Tinainakith; kushtit hitkektatasham hamanm Miahs.

$28 \mathrm{Ka}$ mina tinuhnin hiutsatatu, kunapkitit shakantaih hiwitkainaktatu.

29 Ka kaua wako hilamtainu yokopi shapisiaukas wialahain, kauatita hishamtuks hishaktiu, wah ipinka sikatpama hishamtuks hishaktiu, hetseyu hiwitkauyu, wah Akamkinikainim kapskapsnawinm hiwayatuwuktatashi.

30 Kauana pauwihahnaititaishi hamanm Miāhsna kakamnit, Kaua pawinmakunitatashi uyikashlihpkinih wiwatashpkinih titokanm, hamanm Miahs hiwahyam Akamkinikaikinih, Kapskapsnawithinah, tohshnih siskeisnawithinah, ipalikitpa huhshilikasasham; Kauana immamanahwitatashi.

31 Atka kaua hinashwishapawihnatatasham 
ipnim Haangel, hinashapashapunmitatasham kapish; Kaua Ipna shishaihnin pauwisapaamkaitataishi uipilaptipkinih hahatiapkinih, wah palkaikinih Akamkinikaikinih.

32 Kunki waya ashukanith Fignim taulakina shapasana: mat ikupam ath auyahnaitanih ipna kapapin piskoain, ipna wiapiskowit, katia ath imanaktanih, wako atanm elwehnim hikakamnisham :

33 Kushtit Kapam Kinia himtakna ath aktatashi, kauana ath apasukuanu, wako kia hikakamnisha, wako pishkishpa Kia hiushakalkosham.

34. Ikuin ath hisa, ki naks piim watu hilamnu, atka wako ki himtakt hitsanu laam.

$35 \mathrm{Ki}$ Akamkinikai, wah ki watash ku hilamnah, wah inim tsekinsim watu hiwislamnu. 36 Matu yokopna lahaina, wah yokopnanka Hourna, watu minm naksnim titokanm pasukuasa, wah immamka Haangelm Akamkinikaipa, watu pasukuasi ; ipsiwatnim Natotamsim.

$37 \mathrm{Ka}$ kush Noenm wialahaipa hikushina, kushtit hamanm Miahspim hinashtakakutatasham.

38 Atka iyalpasknim anoktkinikai wialahaipa, hiuyapshina, immamauyakushina, inipt Hiuyanishina, Piulakaikosihna oko Noe haaskika liashpa,

39 Immasukuanaiph iyalpasknim hinashta- 
kakuma, hinashisiaukima laam: Kushtit hamanm Miahs hinashtakakutasham.

40 Atka lapu hipakiaiiku tamanikashpa, kunamana naksna paanpu, naksna pawaunu.

41 Lapu haaiat Hipatutnu tutniwashpa, Kunamana naksna paanpu, naksna pawaunu.

42 Waya imamanakyohoth; atka imam Lord sukuanaipa hourpa ath hitkaikotatasha. 43 Tsalui tahs hama inina inaknikawat hisukuanoka kalapa hourpa hipainoka watqaikawat, kaua ipnolahnanyoka init, Kaua watu manama init tialpash hiutsayoka.

44 Waya imanka imamauyauwanin kunku ath pautsayu: ath sukuanaipa hourpa hipainotatasha hamanm Miahs.

45 Naks hiwatah waiintas ikuin, wapsuh, Kaul kunia ipnim Miohatom pashapanakniktah mamaias, wiashapaptash tsaa;

46 Yiyaunin yokopi waiintas, Ka ipna yohinih Kut papainoaitah ipnim Miohat.

47 Ikuin ath hish, Kunia laam wishanikt pashapanakniktatasha.

48 Matu kaua naks hiwatah mishami waiim. tas; kaua yokopi hitimnatsehnah, mat iku inim Miohat hishausa;

49 Kaua mamaias pauwauiaitah, ipinih hiuyaptah, ipnakotah, Kalaunika hikkauitah ;

50 Ath hisa Kunim waiimtasnim Miohat hipaitatasha, ipnim walahnaipa lahaipa, sukuanaipa hourpa. 
51 Kaua kunia pankauwiyuitatasha, Kolwakishph pasapapatatasha: Kaua Kuna hilalwahwahtatashi, hitit kanptatashi.

\section{WANAHNA XXV.}

KAMKINIKOM Aishniwashnim autsataA tasha Kushka putinwa titamai, Ka imma sapalakauitas hipanpitah, tamasana waukunitash.

2 Pahlo wiwapsuh hiushinah, matu pahlo pipelepipele.

3 Pipelepipele Hipanpitah sapalakauitassim, matu saiau tahsh:

4 Matu wiwapsuh hipanpitah sapalakauitas wah tahsh hikaipa itin.

5 Ki mat tamas hishahshaunah, Kalaunika hipatautaliktah;

$6 \mathrm{Ki}$ mat paah sikatpa hipatiuhnah, wako ath hikakamnisham Tamasam; waya awaukunimth.

7 Kaua yokoma hipatkahohalakaika, sapala. kauitas hipatkawiyakakasaya tasahki,

$8 \mathrm{Ki}$ mat Pipelepipelenim pasana wiwapsuh. na, natsnimth tash, Kia sapalakauitas hilatahaskshi.

9 Kaua wiwapsnhnim pampstakanpshana, pasina watu; Kainanm hilalamkapiku : waya imamain takatamiatath itamiawatoph.

10 Kos hitamiatshaukshinah Ki mat tamas hipainah: Kaua ka Kala immamauyawanin 
hiushinah, immasim paasoatuihsanah tamasam hipitph, Kaua pishkish wakalkin hiutsatah.

11 Kaua imanka pipelepipele titamai hipapainah, pasina Miohat, nashtamahalpanim.

$12 \mathrm{Ki}$ mat hinashsuyatkalaiktah, hinashnah, Ikuin ath hisa, watu ath shukisa.

13 Kunki imamanakyahoth, atka watu sukuanash hiwash, kalapa lahaipa, kalapa hourpa hipaitatasha hamanm Miahs.

14 Atka Akamkinikom aishniwash hiwash Kushka naks hama hiwatah, Kaua ipi hiwihnatatashanah wiatka watashph, Kaua ipnim waiimtas himunah, Kaua ipnashapanaknikaitah wishanikt.

15 Naksna panitah pahat Talents, Kaua naksna panitah lapit, Kaua nakșna naksni; Ka Kush aushinah hahamnim wapsukuawit, Kush hinatsinihnanah; Kaua kunmainih hiwihnanah.

16 Kaua ipnim autsatah pahat Talents, ipi hiluhswiaiiktah hitamiaiiktah yokopnaki kalaunika pauikinih hishapautsatah pahat Talents.

17 Kaua ipnim lapit autsatah, ipinka Kushtit hikotah, Kalaunika pauikinih hishapautsatah lapit.

18 Matu Kaua ipnim autsatah naksni, ipi hipilatah watash, Kaua pawauikaitah Miohatona silpsilp. 
19 Wakepa Kaua immam waiimtasnim Miohat apapaitoktah, Kaua hinashapnitah yokomuna.

$20 \mathrm{Ka}$ ipnim pahat talents autsatah, ipnimkos hipainotah, panahpaikotah pauikinih pahat talents, panah, In Miohat pahatih talents A niwihnanima; waya hakin pauikinih pahat talents A shapautsanya.

21 Kaua Miohat panah, tahsu atam kuya, tahs waiimtas awash, tukutukuh : atka hikutskutsaki atam tukuh Kushaka, wah wako A titilu shapanakniktatasha: Kauo asoatuihnim im Miohatona aishniwash.

22 Kaua ipnimka papainotah, ka ipnim lapit talents autsatah, panah, In Miohat, laptih talents A niwihnanima waya hakin, pauikinih lapit talents A shapautsanya.

23 Kaua Miohatom panah, tahsu atam kuya, tahs waiimtas awash, tukutukuh: atka kikutskutsaki atam tukuh hushaka, wah wako $\mathbf{A}$ titilu sapanakniktatasha: kauo asoatuihnim im Miohatona aishniwash.

24 Kauana ipniınka papainotah, ka ipnim naksni talent autsatah, panah, Miohat, sukuanaisha $A$, piwatqaiktipas hama awash, atka nashapna tupnaitatu $\mathrm{A}$, matu $\mathrm{A}$ watu maua kuna watsastsastatu; nahshpama A sapaamkaitatu, matu watu maua kuna saulakoktatu :

25 Kunki sikausaka A, kunki imana talent 
wawikaishaka watashpa A: wah yoh awa imim.

26 Ki mat ipnim Miohatom pasuyatkalaika, pana ipna, patokash awa, hanimau awa; ikuin asukuanamh nahshapna hinashtupnaitatu, matu watu maua kuna hiwatsastsastatu, nahshapna Hinashapaamkaitatu, matu watu maua kuna hishaulakoktatu,

27 Kaua ina si'psilp inikaitamah taklainawatoph, ka kaua In paitoktah, kaua inim silpsilp inpash witsatah, hishakthinah.

28 Kunki hinashnah, waya anpanith ipna talent, ka ipnim awitsas putimpt talents, ipna aunith ;

29 Ka ipnim autsatatu, ipna panitatasha, Kunki pauikinih ipnim ilahni awitsatatasha; matu taklai ka ipnim saiau autsatatu, ipna panpaitataisha laam patu.

30 Kaua kunia hanimauna waiimtasna atolailakith antka shaktish: Kuna hilalwahwaktatashanki, hitit kanptatashanki.

31 Hipaitatasha hananm miahs ipnim siskeiswithinah, uyikala hautnishpama Haangelhinah, kaua huhshliktatasha ipnim hautnishpa huhshlikasatashpa.

32 Uyikala titokan sapaamkin hiutsatatasha, ipnim inoktkinikaih, kaua hinashapankauyuitatasha Laam, ka Kush sheepna walahnawatom hinashapankauyuitatu sheepna hiyatanin; 
33 Sheepnasim paniktatasha wapsuhpkinikaih, matu hiyatana sahiahkinikaih.

34 Kaua Miohat wapsuhpkinikaipamana hinashtatasha, Kumth yiyaunin Natotapkinih, waya asoth aishniwash, yoh kapam hanianima watashnim anoktkinikai :

35 Atka In hayana, kaua ath hipt panima: kiyausana, kaua ath kush panima: ishtukas waka, sauin ath pashapaasima:

36 Shishmahnot waka, kaua ath pashapashamkima: Komainin waka, kaua ath papainoma: Shaktishpa initpa waka, kaua ath paasoma.

37 Kaua pamstakanptatashi tsitsanishnim, patatashi, Lord, maua kamah aksina hayakiph, kaua kamah shapapoko? Hu ma kiyaunishph, kaua kamah shapakoyoka?

38 Maua kamah Ishtukas aksina, kaua kamah apashapaasoka? hu ma shishmahnot, kaua Kamah pashapashamkitah?

39 Maua kamah aksina Komaiph, hu ma skaktishph initph, kaua kamah papainoyoka?

40 Kaua Miohat hinasmishtakanptatasha, hinashtatasha immnua, Ikuin ath hisa, inaki Kutskutsanihna kinamana inim askapamana ath kushinma, yoh ath Ina Kushinma.

41 Kaua immunanka sahiahkinikaipamana hinashtatasha, wakainin ath Washi, niwihnamth ilatahaskshimaiph alaph, yoh Devilna Haangelhinahna hinashanianima: 
42 Matu In inaki, hayahna sauin ath watu hipt pinima: Kiyausana inaki, sauin ath watu kush pinima:

43 Matu In waka ishtukas inaki, sauin ath watu pashapaasima: Shishmahnot inaka, sauin ath watu pashapashamkima: Komainin, wah shaktishph initph inaki, sauin ath watu papainoma.

44 Kaua immamka pamstakanptatashi, Patatashi, Lord maua kamah aksina imana hayakaph, hu ma kiyauph, hu ma ishtukashna, hu ma shishmahnotph, hu ma komaiph, hu ma shaktishph initph, kaua kamah watu yiyaushina?

45 Kaua hinashsuyatkalaiktatasha, hinashtatasha, Ikuin ath hisa, mat ikupam naksna Kutskutsanihna kinamana watu ath apayiyauna, kunki inanka watu ath payiyaunima.

46 Kaua imma hiwihnatashinki shapopilaptaniwashph, Kunikuaiph animkinikaih : matu tsitsanishnim hiwihnatatashinki wakaswinwashph Kunikuaiph Akamkinikaih.

\section{WANAHNA XXVI.}

T A KAUA Jesus hinashimtahnakia uyika1. la tsekin, kaua Disciplesna hinashna,

2 Wako ath sukuasi lapiskiu simka kaua kia namakatimipnit witsatatashi wauph ; kaua kuna hamana Miahs pahimtakaitataishi shapankatpulitash. 
3 Kaua paamuhna titalamionawat, wah titimanawat, wah titokapama titakash, takashnim talamionawatom initpa, ka ipnim unikshana Caiaphas,

4 Patamaluioshina Jesusna, pisina manmaiih talauyahtimptki anptatashi ipna wapsiaunash. 5 Kaua pisina, ka watu hipitpa, kaina titokan pinahtakanpuyu.

6 Kos Jesus hilakaina Bethanypa, Simonm, Lepernm initpa, .

7 Ki mat naksnim Aiatom papainoya, panahpaikoya Alabasternm (pishwenm) hikaiph itin palh, kaua payakakasanya hushus wahshilikitph.

$8 \mathrm{Ka}$ kaua paksana Disciplesnim, ki mat patananishana, pasana, manama ki kush pau kaisi?

9 Kuh ki palh hitamiatah himakesph, kaua kunki yiyaunash hiuituishna hiutsatah.

$10 \mathrm{Ka}$ kaua Jesus hinashmitsia kaua hinashna, ituain akesksi aiatona? Tahs hikushaka Ina.

11 Atka hahiuit imam ath washi kuniku; matu taklai imam ath washi In miwas.

12 Mat iku Ina tamikishain, Ina silakt kinki palahki hipalhkiaisha.

13 Kunki ka mina wiwatashpa pauyatam. tainaiiktatashi kinki taaishkitki, kunaiitit pauyatamapaikaitataishi. Kinia aiatona kut, ipnimki shapatimipnitash. 
14 Kaua putimwapama wah lapiuwapama naks, unikshana Jedas Iscariot; hinashkioya titalamionawatona,

15 Hinashna, mas ath initatashinm, kaua ath in inahimtaku? Kaua mitaptitki Silvernm sisilpsisilpki piwayanpa.

16 Kaua kunih patamapaiktapaswia.

17 Shapapulamnainim ipahnim wialahain uitpama, .ipnim Disciplespim papainoya ipna, pasana, Mina kamah waun haniaishi hipash?

18 Kaua hinashna, Waya kutath tauyanikash, naksiph hamaph, kunia ath apanu, kush Miohat hihisa, wako inim kut hikakamnishain; wah imim initpa inim Discipleshinah hiptatashi wauna.

19 Ka kush Jesus hinashimtaka Disciplesna kush hipakuya; paniaishana waun.

20 Kulawitpa hipatamayakeka putimwahinah wah lapuwahinah.

21 Hipitpa hinashna, Ikuin ath hisa imam ath washi naks Ina tamapaikash.

22 Kaua uyikala immamatimnahmaina, panaksii pashapnia ipna, Lord ishi In?

23 Kaua Hinashsuyatkalaika, hinashna, ka ipnin kauasim inpshi kiwautakaiph, iptit hitamapaiktatasha Ina.

24 Hamanm Miahs hitsatatasha timash, ka yoh wako timanin ipna: matu wakainin hama, ka ipnim hamana Miahs patamapaikaita- 
taisha! Ka watu hiutsatah yokopi hama, kaua ku tahs.

25 Kauana Judasnim, tamapaikawatom, pamstakanpa, pana, Miohat imatah in wa? kaua pana ipna, wako A im hisa.

26 Kos hipshina, Kaua Jesus ipah hinipa, ipnoyiyauka, pauwitimka, kaua hinatsinya Disciplesna, hinashna Inpukinimth, hipanimth; ki wash inim silakt.

27 Kaua hanaka kikai hinipa, ipnoyiyauka, kaua hinatsinya, hinashna, Koanimth:

$28 \mathrm{Ki}$ wash inim kikat kimtinim piwayanpnim kikat hiwash, shapawalanin ilahniwana patokashwit sapakaikash.

29 Matu ath hisa, In watu hanaka akoaitataisha Vinena timanit, Kosu piamhpa Na-Totam tauyanikashpa, hanaka pikotuatatashi.

30 Kaua hiponipa, kaua Olivehish mahshamka hipakuya.

31 Kaua Jesus hinashna taks sikatpa uyikalo ath Ina sikaunapiktatashinm: atka wako timanin hiwash, Walahnawatona In ashapopsiautatasha, kaua hipuktatashi laam sheep.

$32 \mathrm{Ka}$ kaua In witalahtoku kaua ath inoktianyu Galileeph.

$33 \mathrm{Ki}$ mat Peternm panpstakanpa, pana, Inaki uyikalom A Hiskaunapiktatashi, sauin watu in sikaunapiku A.

34 Kaua Jesusnim pana, Ikuin ath hisa taks 
sikstpa, Kos watu washwashno hinmiai waku wako mitaham imashtaku In wasatki.

$35 \mathrm{Ki}$ mat Peternm pana inaki A tinuhtuatatasha, sauin watu maua Im wasitki inashtaku Kushsim hipatsehna, uyikalo Disciples.

36 Kaua Jesus Discipleshinah hipapaina Gethsemanepa; kaua hinashna; kinu waya tamayakekith kos inoyiyauktasa kunapki.

37 Kaua Peterna wah Zebedeenm mamaiasna lapiuana hinatsnahnana; kaua komais hitimipnia, hitimnatsinina.

38 Kaua hinashna kia timnatsinintamausa, kushka tinukin: Kinu witsath, ka namanakiohos.

39 Kaua hipakuya kimtam kuph, kaua hitqailushtakailika, ipnoyiyauka, hihina, hiiya Tota Kum Im kinia hikaina ashapakokaunamh, sauin watu inim timiuki, imimsim tinniuki.

40 Kaua Disciplesna hinashkioya, ishimat hitautasi, kaua Peterna pana, imat! watu im imanakiohotash ina, naksnipa hourpa?

41 Inamanakiohoth, Imamoyiyaukith, kainapam hiyalatatasham: Wakaswinmsin kesknash, matu silakt ilat.

42 Kaua hanaka hikuya ipnoyiyauktana, hihina, hiiya Tota, kuh ki hikai hikokaunah inakoyai, sauin imimsim timiuki.

43 Kaua Disciplesna hanaka hinashpainoya, 
ishimat hanaka hitautasi; mat iku silu sininish aushina.

44 Kaua hinashniwihnana, hanaka hikuya mitaham, mitaham ipnoyiyauktana, yohtit hitsehna:

45 Kaua hanaka Disciplesna hinashpainoya, hinashna imat pinimshi? imat suqaisi ? matu wako hitsasa yokopi kut. Wako hamanm Miahs tamapaikin pipatokashnim ipshusph.

46 Hohalakaikith Ka pakush: Ahakin, yoh hiwahyam, ka ipnim Ina hitamapaiksha.

47 Kos hitsehsana, Kaua hipaina Judas putimwapama wah lapiuwapama, ki mat ilahninm patuihsinm, walswinin hatsuhinah, titalamionawatopkinih titakashkinih.

48 Wako ata Himtakawat hinashna immuna, kah kunia in ahimkatsaku suata yoh hiwash: Kunia ath apanpu.

49 Kunki lamlamat Jesusna pauwaukunia, pahimkatsaka, pana, Miohat, aishin.

50 Kaua Jesusnim Pana sikstua ituph kuma! Kauana puyakioshana Jesusna, paanpshana.

$51 \mathrm{Ki}$ mat Naks Jesusnim wiatwa wals hitkanipa, talamionawatoin waiimtasna pawauwiana, matsaiyu pushupanya.

52 Kaua Jesusnim pana wals itatashph ipaitaih wals: Ka imma wals inahtakanptanih, imma walaski hitkaukaitatashi.

$53 \mathrm{Kum}$ in sukuanamh, kuh In inoyiyauk- 
tah Na-Totamph, kaua lamlamat hinash waiimtatamh putamaham wah lapaham Legions Haangel ?

54 Matu kaua minmaii yokopi timash hitsanah? Kala tsanash hiwash.

55 Kunaititpa hourpa Jesus hinashna pauwatashna, inat ina takakioshinm kushka piwatqaiktipasna, wals winin hatsuhinah? Mat ath wiapishaka Templepa (initpa) wialahaipa ath wiatamtaisaka, sikaunai; sauin ath watu ina inpshinmka.

56 Matu kinm wiakunm patsananima imatsinpuna timash. Kauana Laam Disciplesnim paulalihnapika ipna.

57 Kaua ka immam paanpshana Jesusna, immam panahnasana ipna Caiaphasph, takash talamionawatoph, kaua paamhnoshana Titimanawatom wah titakashnim.

$58 \mathrm{Ki}$ mat Peternm patuihna ipna wiatka, takash talamionawatom inith paasoatuihna, waiimtas hinatsipaiya, hahnash lamtai kut.

59 Kaua titalamionawatom, wah uyikala titakashnim, wah uyikalanm mimiohatom papawishana shapanahmishamtash Jesusna wapsiaunash :

60 Sauin watu paiyaksana: inaki ilahni inahmishamtash huyapaina, sauin keyups. Kalaunika lapu inahmishmat hipapaina.

61 Hipahina, ki hisaka, In wash Godna Tem- 
ple nikaskilkash, kaua kuniatita laulimkash wash mitaskiupa.

62 Kaua hiushakaika takash talamionawat, pana ipna, Manama watu A nashmishtakanpsha? Manama hitamapaikshi A ?

$63 \mathrm{Ki}$ mat Jesus shauish hiutsaya. Kauana takashnim taiamionawatom pana, shapatsehsa A Tinuhshimaiki Godki, imatamapaih, wat wa yokopi Christ Godnim Miahs?

64 Kaua Jesusnim pana, yoko A wako hisa: Sauin kama himtaks, anmakunitatasha $\mathrm{A}$ hamanm Miahs, hiwahyam ipalikitpa, Kapskapsnawinm wapsuhpkinikai wasulishtakin.

65 Kaua takash talamionawat shamh hinkahtatka, hihina, awah, ipnanahkiyusha; wah ituna uikinih tamapaiktash apawishi, wako ath amsiaishi ipnanahkiyut.

66 Wah manama ima nakshi? Ki mat pamstakanpshana, pasana wapsiaunashu hiwash.

67 Kaua patulsaishana mash tai, paptashina, tatoshmam him paptaishina,

68 Pasina, Christ awa, waya anashtamapaih; ishinm iptasha.

69 Kuna Peter huhshalika; Ki mat naksnim timainim papainoya, pana matu $A$, im auyatawashaka Jesusna Galilee puna.

$70 \mathrm{Ki}$ mat ipnashtaka uyikalaph hihina watu, kurn ituatash hisam.

71 Kaua haatkika, Kaua naksnim pahna 
ipna, hinashna hohalikith, kat kinm pauyarwashaka Jesusna Nazarethpuna.

72 Ipnanahkiyutki Hanaka ipnashtaka, hinashna watu ashukisha kunia hamana.

73 Miwaspa Hohalikitkinih pasina Peterna, 1kuin kunama awash; atka im timnim $\mathrm{A}$ hitamapaiksha.

74 Kaua ilahninaham palunika, hinashna watu ashukisa yokopna hamana. Kauatita hinmia washwashno.

75 Kauana Peternm patimipnanya Jesusna tsekin, yoh ka pana ipna, kos watu washwashno hinmiu, kaua wako mitaham imashtakin awitsayu inwasatki. Kaua haatkika, komais kaua hiwina.

\section{WANAHNA XXVII.}

AYUI uyikalanm titalamionawatom, wah 1 titakashnim patamaluioshina Jesusna wapsiaunash.

2 Kaua pulatpashana, panahnasana, miohatona, Pontius Pilatena panahpaikoshana.

3 Ka kaua Judas tamapaikawat hisukuana ata wako tamaluioin ipna, kaua ipnatanania, hinatsnitoka. Titalamionawaton a wah titakashna, yokopi mitaptit Silvernm sisilpsisilp, 4 Hinashna, kia Kapshish kushaka, ata kapshishwiaina kikat ahimtakaishaka. Ki mat immam pasina, mishah yoh nunim kutash? kala imsiwatnim kutash. 
5 Yokopna Silvernm sisilpsisilpna payakaka Templepa, kaua haatkika, kaua hikuya ipnolaukaka.

6 Kaua titalamionawat hipanipa yokopi Silvernm sisilpsisilp, kaua pisina watu hiwash inikash Treasuryph, kala kikanm tamaiat hiwash.

7 Kunki pitamaluishina, kaua patamiaishina shitahnim hikai haniawatona watash, istukashain wiatamikash.

8 Kunki Kunia Watashna pauyanima kikatin watash oko taksiph.

9 Kaua patsananima Jeremyna imatsinpuna tsekin, yoh ka hihina, atkic hipanipa yokopi mitaptit Silvernın sisilpsisilp shapatsanishki itamaiat, ka kunapki pashapatsana Israel kakanm pamanm;

10 Kaua kunki patamiaishina shitahnim hikai haniawatona watashph, Lordnim tamaluitki.

11 Kaua Jesus hiushatuna miohatom inoktkinikai: Kaua miohatom pashapnia, pana wat wa Jewsnim miohat? kaua Jesusnim pana. yoko A tseksa.

12 Inaki titalamionawatom wah titakashnim patamapaikaishana ipna patupama, sauin watu hinashmishtakanpa.

13 Kunki Pilatenim pana, wat A nash. mitsisha patupama yoh A hitamapaikai. shi? 
$14 \mathrm{Ki}$ mat ipnanka watu pamstakanpa; Kalaunika miohatom patsiwaitamauna ipna.

15 Wiapitpa miohatom pankapkuikainihna naks walatpishpama, ka minia titokanm pa. yiyaunanihna.

16 Ata aushina naks sikauish walatpin, unik. shana Barabbas.

17 Ka kaua piamhnin hiushina, kaua Pilate hinashapnia, so miniku kapamah nikapkuikaisha? Barabbas, hu ma Jesus Ka ipi wanikin Christ?

18 Mat iku hinashsukuanaishana, katamnotki panahpaikshi ipna.

19 Ka kaua Shapatkukinwashpa huhshalika, Ki mat Iwapnapim Pashapatatktana, pana wat mat yokopna tsanishna hamana manama pakiyu; atka taks Komais inatananishaka hiwakitpa ipnimki.

$20 \mathrm{Matu}$ titalanionawatom wah titakashnim pashapayiyausana pauwatashna Barabbas, Kana Jesus pashapopsiauna.

21 Kaua miohat Hinashapnia, hinashna minikuna kapamah nikapkuikanih ki lapu? Ki mat pasina Barabbas.

22 Kaua hinashna matu manamah kaua in Jesusna kutatasha, Ka ipi wanikin Christ? Kaua uyikalanm pasina Nikatpulitashu hiwash.

23 Kaua miohat hinashna matu ituph kap- 
shish hikushaka? Kimat uikinih hipatuhna, pașina aulashapankatpulih.

24 Ka kaua hinashahna imat watu tiakalkash hiushi, kala uikinih hituksi, kaua kush hinipa, kaua pauwatashnim inoktkinikai hiwapaika, hinashna, kapshiswiai witsasha kinih tsanishnim kikatkinih: Imosiwatnim kut ath witsashi.

$25 \mathrm{Ki}$ mat uyikalanm Titokanm pamstakanpshina, pasina, A, kuh nuna wah uikinih nuın mamaiasna hinashwiashamitsitatasha kinm kikanm.

26 Kaua hinashnikapkuikanya immuna Barabbas: Kaua Jesusna pawauiana, kaua hinatsinia ipi nakatpulitash.

27 Kaua miohatom piwapsiaunatom panahnasana Jesusna miohatom initph, kaua paamhnoshina naks inakniknim.

28 Kaua pawahwaimkshana ipna, kaua ilpilp siskan pashapasiskanishana.

29 Kaua Shapashitshitnin shishnim, pashapashushi-hana, kaua Toko pashapanpshana: Kaua pahitimlikoshana, popilaptaisana, pasina Judeapum Miohat, Aishin!

30 Kaua patulsashana, pauwauiaishana hu. shus yokopnaki tokoki.

$31 \mathrm{Ka}$ kaua wako watsu popilaptaisana, kaua yokopi siskan paukahwaimkshana, kaua ipnimnihki shamahki pashapashamkishana, kaua panahnasana nikatpultash. 
32 Ka kaua imma hipaata, ki mat paktsasana naksna hamana, Cyrenepu, uniksarı Simon: Kaua taklai kunia pashapashapshana ipnimnikatpulitash.

33 Ka kaua nakspa watashpa hipapaina, Golgothapa, tamanin Athushusin watash.

34 Kaua kuna panishana ipna Vinegar shapatuanin makaski shapakutash: Ka kaua pahimkashuka, ki mat watu ipnakoya.

35 Kauana pankatpulishana ipna, shamh patu patakanaishana, shapinauyaii; kunki pashapatsanaishana imatsinpunm tsokaph, Ina shamh patu hipatakananya immamain; siskan hipatakananya shapinauitashki ;

36 Kaua hitamayakshina, ipna pulahsina;

37 Ipna tamapaikt patimananya, KI HIWASH JESUS JUDEAPUM - MIOHAT, Kaua hushusnim kunmakam pashapalishtakanya.

38 Lapiuaki piwatqaiktipaski pashapankatpulituana ipna ; naks wapsuhpkinikaipa naks sahiahpkinika.

39 Kiaiikitpkinih pothtalaulakishina, pau. wautakioshana,

40 Pasina, im A Templena ankaskilktah, kaua mitaskiupa alaulakitoktah, kauo Wakas imanih. Tsalui Godnim Miahs awash kauo. tamikim nikatpulitkinih.

41 Kushtit Titalamionawatom wah titima: 
nawatom wah titakashnim, patalaptaisina, pisina,

42 Nahshapnasim wakas pihanitipas, matu ipini watu wakas ipnanitas. Ikuin hiwatah Israel Miohat, kaua hitamiktamh, nikatpulitkinih kaua kia apamsukuanaktah.

43 Atka tukalukt oka Godiph; tsalui ipnim otah ki, kunanm hihitoktamh ki, atka hisana Godnim Miahs wash.

44 Piwatqaiktipasnim, Ka kuniin nikatpulituanin hiushina, imınamka patalaptaisina.

45 Wilaksipkinih Hourpkinih pashaktioma uyikashlihna watashna oko kuisipa Hourpa.

46 Kaua kuisipa hourpa Jesus hitsehna kapish, Eli, Eli, lama Sabachthani? Tamanin hiwash, Inim God, Inim God, manama Ina kalaunapiksham?

47 Hohalakitpkinih Pamsishina, Kaua pasina, ata Eliasna pamusa.

48 Kaua naks lamlamat Hulakaika, Spunge hinapa, kaua Vinegarpa iptalukika, Kaua hatsuki pasuskaka, kaua pashapasuhsuhna ipna.

49 Matu imma hisina, itu akesksa, kos kia sukuatatashi, kunanm Eliasnim hihitoktatasham.

50 Kaua hanaka Jesus kapish hitsehna, kauana hitkaukaina.

$51 \mathrm{Ki}$ mat Templem Veil hitkatatka, tush- 
kinih oko kith; watash hitkatuwuka, wah tilel hwaiitimka.

52 Wah titamikash hiwiwayahalpa, kaua ilahninm tsitsanishnim tinuhnishpkinih witalaht autsaya.

$53 \mathrm{Ka}$ kaua Ipi hiutalahtoka Kaua immanka tamikashpkinih pautalahtokima, Hautnishpa tauyanikashpa paasoya, ilahniph immamashapahna.

$54 \mathrm{Ka}$ kaua yokopi Centurion inaknikthinah Jesusna walahnat, paksina watashna wayatuwukitph wah uyikalaph wiakutph, kaua ata hipaskautmauna, pisina, Ikuin àta hiwaka Godniin Miahs.

55 Wiatkinih ilahninm haaiatom pulaksina, ka immamtit Galileepkinih patuihsina Jesusna pauyayiyausana Ipna.

56 Mary Magdalene, wah Mary Jamesnim wah Josesnim pika, wah Zebedeenm mamaiaspim pika.

57 Wako hikulawitsana, ki mat kuishnim hamanm hipaina Arimatheapkinih, uniksana Joseph, ipinka hiwaka Jesusnim Disciple. 58 Ipnim pakioya Pilatena, Panahmuna Jesusnim Silakt Kaua Pilatenim hinashapania Silakt ipnimki.

59 Kaua Josephnim panakahnanya silakt, kaua pasapalkolikanya Linenm Kåihkaihki tahaiki,

$60 \mathrm{Ki}$ mat panika ipnimnikaiph Kimtiph 
Tombph, (tamikash) pishwepa ata ipnanioin awka: himakesli pishweki patamakilka, Kaua hipaskilina.

61 Tamikashnimakam hitamayahshina, Mary Magdalene, wah yokopi naks Mary.

62 Ipnauyauwanashnim lahaipa katatpa lahaipa, paamhnoshina Pilatena titalamionawat. om wah Phariseepum,

63 Pasina, Miohat, nu amsiaisheka yokopna shapapelenatona tsekin, kos wakas hiwaka kaau hitatu, mitaskiupa kaua In witalahtoktatasha.

64 Waya uimitatipa wialahaipa ashapatiauiswith kunia Sepulchrena (tamikash;) kaina ipnim Disciples sikatpa hipainu, kaua paanpshanu, kaua titokana pasanu, wako Tinuhnishkinih hitutalahtoka ipi : ‘unki halakinmam mishaminın pakatoshku anakonmana.

65 Kaua Pilate Hinashna, Matu walahnawat ath washi: Kutathku apashapasapantaku imam wapsukuawitki ath apashapulahnu.

66 Kauo hipakuyu, Tiauis Papakilkshana Sepulchrena yokopna pishwena pasapalishtakshana Sealki, pashapulakshana.

\section{WANAHNA XXVIII.}

W $\begin{gathered}\text { AKO halahpawit Hilaamtaina, Kaua } \\ \text { taklai Week Huuiya, hauwapa Hipa- }\end{gathered}$ paina Mary Magdalene, wah yokopi naks Mary Sepulchrena (tamikash) palitasana. 
2 Mat ata wako watash Hiwayatuwuka Kapish: Atka Akamkinikaikinih Lordnim angelpim Hitkekima, Yokopna Pishwena Patamaskalikapika pishkishkinih, Kaua Kuna huhshilikasaiya.

3 Mashtai awak Ka Kush hitkasaioho, shamh awaka haihai kushka maka:

4 Walahnawat hipahpipipna sikauki Kunia, kushka tinuhnin hipautsaya.

5 Kaua yokopi angel hitsehna, haaiatona hinashna, Wat mat sikaunanih: Wako In sukuasa, Jesusna ath apawiaiikshi, ka ipi nikatpulin hiwaka.

6 Saiau hiwash; wako hiutalahtoka, ka kush oko hisaka. Kumth, waya ahnanith Lordna tamalikitpama.

7 Kauo hamtis inahwulakaikith, ipnim Disciplesna atamtaitath, wako ata hiutalahtoks Tinuhnishpkinih Ipi, Ipinkos hikusha Galilee; kuna ath aktatashi Ipna: Mitsimth, wako ath himtaksha.

8 Kunihnih Tamikashpkinih hipakuya, Sikauki wah Aishki hipanahwulakaika ipnim Disciplesna tamtainash.

9 Kos Ipnim Disciplesna panahwulakioshana, ki mat Jesus hinashwaukunia, hinashna Aishith uyikalo. Ki mat pakioshana, Ahwe Paanpaishana, kaua Ipnimphki immamoyiyaukshina.

10 Kaud Jesus hinashna wat mat sikauna- 
nih: waya Atamtainatamth Ina Askama, Galileeph ath kushi, kuna Ina haktatashi.

11 Wako hipakuya Imma, kaua ata immanka tatoshma Walahnawat panahwulakaika tauyanikashph, titalamionawatona pashapasukuatasana uyikalapama ka kush pakseka.

12 Kunki paamhsina titakashinah, kaua Hitamaluishina, Kaua ilahni sisilpsisilp panishana piwapsiaunatona,

13 Pasina, waya tamtainaiikith, Kala ipnim Disciplespim sikatpa papainoshna kaua paanpshana yokopna num taukanam.

14 Tsalui miohatom pamsitatasha kinia tsekin, kaua ipna aulatapaitataishi, kaua imuna wakas ath pashapautsayu.

15 Kaua yokopna Sisilpsisilpna hipanipa, ka kush himtakin hipautsaya, kush hipakuya: Kunkitita tsehki Judeapu hiuyatseksi oko taksiph.

16 Kaua putimwa wah naks Disciples hipakuya Galileeph, naksiph mahshamka, ka kuna Jesus hinashimtaka.

17 Kaua paksina ipna, kaua immamoyiyaukshina ipnimphki; matu tatoshmam patalauiksina.

18 Kaua Jesus hinashkioya, hinashtanueya, hinashna, wako Ina hinima uyikala Akamkinikaipama wah uyikala watashpama Kapskapsnawit.

19 Kunki wiwihnath, awitaaishkaiikith, 
uyikashliph, wiwatashph, Awibaptainaiikith immuna Pishitpim wanikitph, wah Miahspim. Wanikitph, Wah Holy Ghostnim wanikitph; 20 Apashapanakniku immuna ki wiatsekin laam, kapamah Kala wiahimtaka: Waya mitsimth, In ath Wiatwatatasha Kunku, oko watashnim Lamtaiph. Amen. 









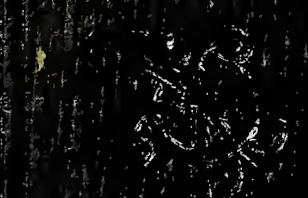

$4 y^{4}$
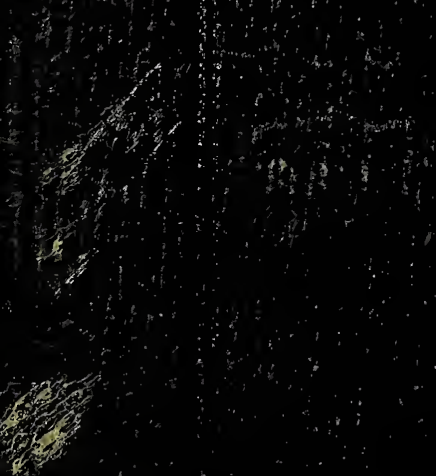

Fre

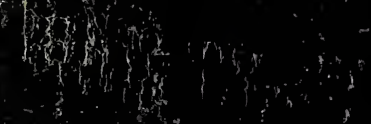

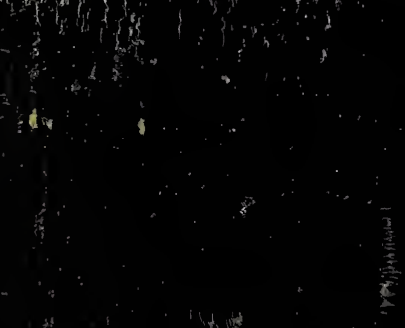

te

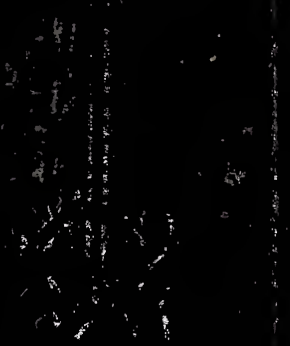

